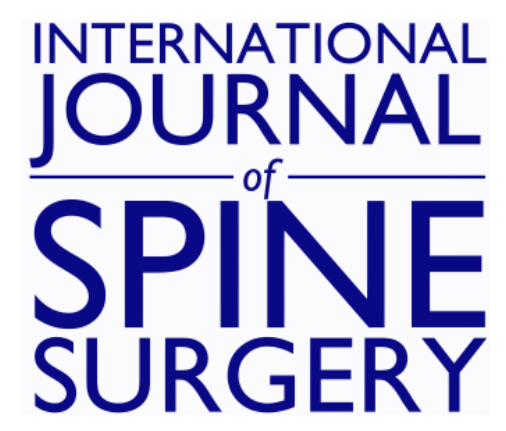

\title{
Surgical Treatment Strategies for High-Grade Spondylolisthesis: A Systematic Review
}

Peter G. Passias, Caroline E. Poorman, Sun Yang, Anthony J. Boniello, Cyrus M. Jalai, Nancy Worley and Virginie Lafage

Int J Spine Surg 2015, 9 ()

doi: https://doi.org/10.14444/2050

http://ijssurgery.com/content/9/50

This information is current as of April 26, 2023.

Email Alerts Receive free email-alerts when new articles cite this article. Sign up at:

http://ijssurgery.com/alerts

The International Journal of Soing Surgerih http://ijssurgery.com/ by guest on April 26, 2 2397 Waterbury Circle, Suite 1,

Aurora, IL 60504, Phone: +1-630-375-1432

(C) 2015 ISASS. All Rights Reserved. 


\section{Surgical Treatment Strategies for High-Grade Spondylolisthesis: A Systematic Review}

Peter G. Passias, MD, Caroline E. Poorman, BA, Sun Yang, BA, Anthony J. Boniello, BS, Cyrus M. Jalai, BA, Nancy Worley, MS, Virginie Lafage, PhD

Division of Spinal Surgery, Department of Orthopaedic Surgery, NYU Medical Center Hospital for Joint Diseases, NYU School of Medicine, New York City, New York, USA

\section{Abstract}

Background

HGS is a severe deformity most commonly affecting L5-S1 vertebral segment. Treatment available for HGS includes a range of different surgical options: full or partial reduction of translation and/or abnormal alignment and in situ fusion with or without decompression. Various instrumented or non-instrumented constructs are available, and surgical approach varies from anterior/posterior to combined depending on surgeon preference and experience. The aim of this systematic review was to review the literature on lumbosacral high-grade spondylolisthesis (HGS), identify patients at risk for progression to higher-grade slip and evaluate various surgical strategies to report on complications and radiographic and clinical outcomes.

Methods

Systematic search of PubMed, Cochrane and Google Scholar for papers relevant to HGS was performed. 19 articles were included after title, abstract, and full-text review and grouped to analyze baseline radiographic parameters and the effect of surgical approach, instrumentation, reduction and decompression on patient radiographic and clinical outcomes.

Results

There is a lack of high-quality studies pertaining to surgical treatment for HGS, and a majority of included papers were Level III or IV based on the JBJS Levels of Evidence Criteria.

\section{Conclusions}

Surgical treatment for HGS can vary depending on patient age. There is strong evidence of an association between increased pelvic incidence (PI) and presence of HGS and moderately strong evidence that patients with unbalanced pelvis can benefit from correction of lumbopelvic parameters with partial reduction. Surgeons need to weigh the benefits of fixing the deformity with the risks of potential complications, assessing patient satisfaction as well as their understanding of the possible complications. However, further research is necessary to make more definitive conclusions on surgical treatment guidelines for HGS.

Level of Evidence

II

KEYWORDS: HIGH-GRADE SPONDYLOLISTHESIS, SURGICAL TREATMENT, INSTRUMENTATION, REDUCTION, DECOMPRESSION, OUTCOME, COMPARATIVE OUTCOME, COMPLICATION

VOLUME 9 ARTICLE 50 DOI: 10.14444/2050

\section{Introduction}

Spondylolisthesis refers to anterior or posterior displacement of adjacent vertebrae. The Wiltse classification divides spondylolisthesis based on anatomic presentation and etiology: isthmic, dysplastic, degenerative, traumatic, and pathological. ${ }^{1}$ Symptoms include low back pain, radicular neuropathy, or me- chanical instability, and deformity as the "listhetic posture" of hyperlordosis cephald to the LS deformity, but many patients remain asymptomatic. The incidence of spondylolisthesis in the general population is $4-8 \%$, with isthmic being the most common. ${ }^{2}$ In 1994, Bartolozzi and Marchetti developed a separate classification system that introduced "developmental spondylolisthesis," which combined dysplas- 
tic and lytic etiology. This category highlights that many high-grade slips result from multiple stress fractures with a developmental etiology whereas lower grade slips commonly exhibit a degenerative etiology. ${ }^{3}$ Both dysplastic and isthmic components can occur simultaneously; therefore, it is difficult to deduce true slip incidence. ${ }^{4}$

Slips greater than $50 \%$ are defined as high-grade spondylolisthesis (HGS), (see Figure 1) which accounts for $1 \%$ of spondylolisthesis patients, but constitutes a more serious pathology. ${ }^{5}$ Patients who progress to HGS are frequently symptomatic with back pain, radicular symptoms from nerve root irritation and postural deformities such as compensatory lumbar hyperlordosis for focal kyphosis. Surgical intervention is generally favored over non-operative management for patients with symptomatic HGS to halt deformity progression and provide symptom relief. However, no consensus for optimal surgical modality exists. Historically, surgeries performed for HGS mimicked those utilized for low-grade spondylolisthesis such as in situ uninstrumented posterior fusions. ${ }^{1}$ However, due to concerns over further progression and pseudarthrosis, particularly in adults, coupled with technological and surgical advancements, surgeons now favor instrumented fusions.

Current treatment options for HGS vary greatly from extent of reduction and/or instrumentation and interbody support use to anterior or posterior-based approaches with or without decompression. Existing literature on surgical approaches is limited to mostly

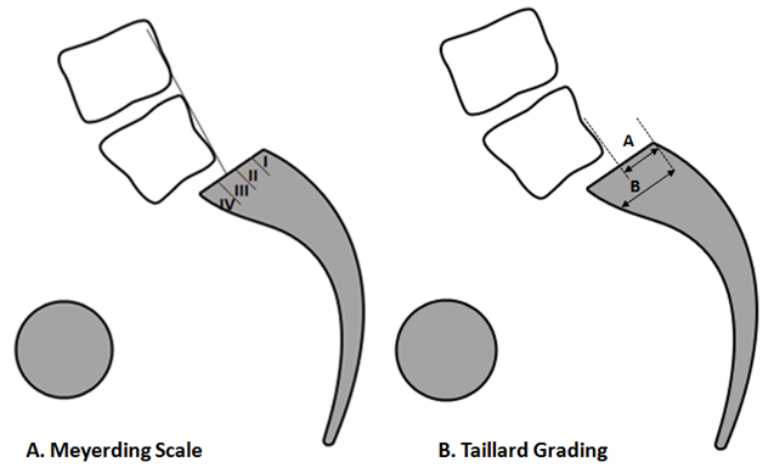

Fig. 1. Measurement of slip angle. Meyerding scale divides the lower vertebral body into quarters and a grade of I to IV assigned respectively. Taillard grading calculates the percentage of the slip from the lower vertebra. small retrospective and uncontrolled case series, and high-quality controlled comparative series on HGS necessary for definitive conclusions are unavailable. ${ }^{2}$ This systematic review attempts to compile best available recent evidence for risk for progression, surgical approach and radiographic and clinical outcomes to formulate a comprehensive review of the safest and most effective surgical options for HGS.

\section{Materials and Methods}

\section{Literature Search}

We performed a systematic search of PubMed, the Cochrane Library, and Google Scholar for literature published from January 2002 - December 2014. The search was limited to human studies and articles published in English using search terms "isthmic, developmental, high-grade, and severe spondylolisthesis." Inclusion and exclusion criteria are listed in Table 1 . Articles were chosen by title, abstract and full article reviews. If studies were from the same hospital with overlapping patient populations, the most comprehensive paper was chosen and others were excluded unless they provided different results or insight for a certain question.

\section{Data Analysis}

For each clinical study data on surgical approach, instrumentation type and extent, reduction procedure, and decompression procedure were extracted and analyzed based on clinical and/or radiographic outcomes. Additionally, radiographic parameters considered in the studies and used to evaluate surgical outcomes included pelvic incidence (PI), sacral slope (SS), pelvic tilt (PT), and slip angle (Figure 2).

\section{Strength of Body of Literature}

Each included study was rated by two independent observers for its Level of Evidence (LOE) based on the Journal of Bone \& Joint Surgery, American Volume guidelines (Table 2). ${ }^{3}$ This literature review studied 14 clinical studies - three LOE II, five LOE III and six LOE IV-and six radiographic studies - three LOE II, one LOE III and two LOE IV. No LOE I studies on HGS treatment were found. A majority of current literature consisted of retrospective case and comparative studies. 


\section{Clinical Recommendations and}

\section{Consensus Statement}

Clinical recommendations or consensus statements were made where appropriate using the GRADE/ $\mathrm{AHRQ}^{4,5}$ approach that deliberately separates the

\begin{tabular}{|c|c|c|}
\hline $\begin{array}{l}\text { Study Compo- } \\
\text { nent }\end{array}$ & Inclusion & Exclusion \\
\hline Subjects & $\begin{array}{l}\text { Patients with high-grade } \\
\text { isthmic spondylolisthesis }\end{array}$ & $\begin{array}{r}\bullet \mathrm{N}<10 \text { patients } \\
\text { - Spondylolisthesis caused } \\
\text { by underlying disorder } \\
\text { - Low-grade, degenerative or } \\
\text { dysplatic spondylolisthesis } \\
\bullet \text { Infection } \\
\bullet \text { Tumor }\end{array}$ \\
\hline Study Design & $\begin{array}{r}\text { - Randomized and non- } \\
\text { randomized controlled tri- } \\
\text { als } \\
\text { - Retrospective Reviews } \\
\text { - Radiographic studies } \\
\text { evaluating subject out- } \\
\text { come }\end{array}$ & $\begin{array}{r}\bullet \text { Cadaver studies } \\
\bullet \text { Case reports } \\
\text { - Meeting abstracts/ lectures } \\
\text { - Biomechanical analyses } \\
\text { - Studies evaluating classifi- } \\
\text { cation systems } \\
\text { - Studies not written in Eng- } \\
\text { lish } \\
\text { - }<2 \text {-yr follow-up (for clini- } \\
\text { cal studies) } \\
\text { - Studies before } 2002\end{array}$ \\
\hline Intervention & \multicolumn{2}{|c|}{ May or may not undergo operative treatment } \\
\hline $\begin{array}{l}\text { Radiographical } \\
\text { measures }\end{array}$ & $\begin{array}{r}\bullet \text { Percent slip } \\
\bullet \text { Pelvic incidence } \\
\bullet \text { Sacral slope } \\
\bullet \text { Lordosis/kyphosis } \\
\text { - Level of spondylolisthe- } \\
\text { sis }\end{array}$ & NA \\
\hline Clinical factors & $\begin{array}{r}\text { - Complications } \\
\text { - Comorbidities } \\
\text { - Neurological status }\end{array}$ & NA \\
\hline $\begin{array}{l}\text { Surgical fac- } \\
\text { tors }\end{array}$ & $\begin{array}{l}\text { - Levels of fusion } \\
\text { - Instrumentation } \\
\text { - Decompression } \\
\text { - Approach }\end{array}$ & NA \\
\hline Outcomes & $\begin{array}{r}- \text { Reduction of slip angle } \\
- \text { Radiological outcome } \\
\bullet \text { Functional scores } \\
\bullet \text { Complications }\end{array}$ & NA \\
\hline
\end{tabular}

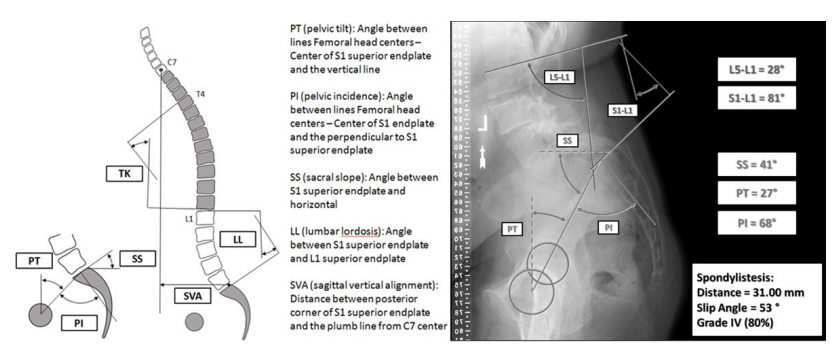

Fig. 2. Left: Illustration of the spinopelvic parameters; Right: CT image of patient with HGS illustrating radiographic parameters (lumbar lordosis, pelvic tilt, sacral slope and pelvic incidence.
Table 2. Levels of Evidence for Primary Research Question.

\begin{tabular}{|c|c|c|c|c|}
\hline & \multicolumn{4}{|c|}{ Types of Studies } \\
\hline & $\begin{array}{r}\text { Therapeutic } \\
\text { Studies Investi- } \\
\text { gating the Re- } \\
\text { sults of Treat- } \\
\text { ment }\end{array}$ & $\begin{array}{r}\text { Prognostic } \\
\text { Studies In- } \\
\text { vestigating } \\
\text { the Outcome } \\
\text { of Disease }\end{array}$ & $\begin{array}{r}\text { Diagnostic } \\
\text { Studies Investi- } \\
\text { gating a Diag- } \\
\text { nostic Test }\end{array}$ & $\begin{array}{r}\text { Economic } \\
\text { and Deci- } \\
\text { sion Analy- } \\
\text { ses Develop- } \\
\text { ing an Eco- } \\
\text { nomic or } \\
\text { Decision } \\
\text { Model }\end{array}$ \\
\hline $\begin{array}{l}\text { Level } \\
\text { I }\end{array}$ & $\begin{array}{r}\text { 1. Randomized } \\
\text { controlled trial } \\
\text { a. Significant dif- } \\
\text { ference } \\
\text { b. No significant } \\
\text { difference but } \\
\text { narrow confi- } \\
\text { dence intervals } \\
\text { 2. Systematic re- } \\
\text { view }{ }^{2} \text { of Level-I } \\
\text { randomized con- } \\
\text { trolled trials } \\
\text { (studies were ho- } \\
\text { mogeneous) }\end{array}$ & $\begin{array}{r}\text { 1. Prospec- } \\
\text { tive study }{ }^{1} \\
\text { 2. Systematic } \\
\text { review }{ }^{2} \text { of } \\
\text { Level-I stud- } \\
\text { ies }\end{array}$ & $\begin{array}{r}\text { 1. Testing of pre- } \\
\text { viously devel- } \\
\text { oped diagnostic } \\
\text { criteria in series } \\
\text { of consecutive } \\
\text { patients (with } \\
\text { universally ap- } \\
\text { plied reference } \\
\text { "gold" standard) } \\
\text { 2. Systematic re- } \\
\text { view }{ }^{2} \text { of Level-I } \\
\text { studies }\end{array}$ & $\begin{array}{r}\text { 1. Clinically } \\
\text { sensible } \\
\text { costs and al- } \\
\text { ternatives; } \\
\text { values ob- } \\
\text { tained from } \\
\text { many stud- } \\
\text { ies; multi- } \\
\text { way sensitiv- } \\
\text { ity analyses } \\
\text { 2. Systemat- } \\
\text { ic review }{ }^{2} \text { of } \\
\text { Level-I stud- } \\
\text { ies }\end{array}$ \\
\hline $\begin{array}{l}\text { Level } \\
\text { II }\end{array}$ & $\begin{array}{r}\text { 1. Prospective } \\
\text { cohort study } 3 \\
2 . \text { Poor-quality } \\
\text { randomized con- } \\
\text { trolled trial (e.g., } \\
<80 \% \text { follow-up) } \\
\text { 3. Systematic re- } \\
\text { view }{ }^{2} \\
\text { a. Level-II stud- } \\
\text { ies } \\
\text { b. nonhomoge- } \\
\text { neous Level-I } \\
\text { studies }\end{array}$ & $\begin{array}{r}\text { 1. Retrospec- } \\
\text { tive study } \\
2 . \text { Study of } \\
\text { untreated } \\
\text { controls from } \\
\text { a previous } \\
\text { randomized } \\
\text { controlled tri- } \\
\text { al } \\
\text { 3. Systematic } \\
\text { review of } \\
\text { Level-II stud- } \\
\text { ies }\end{array}$ & $\begin{array}{l}\text { 1. Development } \\
\text { of diagnostic cri- } \\
\text { teria on basis of } \\
\text { consective pa- } \\
\text { tients (with uni- } \\
\text { versally applied } \\
\text { reference "gold" } \\
\text { standard) } \\
\text { 2. Systematic re- } \\
\text { view }{ }^{2} \text { of Level-II } \\
\text { studies }\end{array}$ & $\begin{array}{r}\text { 1. Clinically } \\
\text { sensible } \\
\text { costs and al- } \\
\text { ternatives; } \\
\text { values ob- } \\
\text { tained from } \\
\text { many stud- } \\
\text { ies; multi- } \\
\text { way sensitiv- } \\
\text { ity analyses } \\
\text { 2. Systemat- } \\
\text { ic review }{ }^{2} \text { of } \\
\text { Level-II } \\
\text { studies }\end{array}$ \\
\hline $\begin{array}{l}\text { Level } \\
\text { III }\end{array}$ & $\begin{array}{r}\text { 1. Case-control } \\
\text { study } \\
\text { 2. Retrospective } \\
\text { cohort study } \\
\text { 3. Systematic re- } \\
\text { view }{ }^{2} \text { of Level- } \\
\text { III studies }\end{array}$ & & $\begin{array}{r}\text { 1. Study of non- } \\
\text { consecutive pa- } \\
\text { tients (no consis- } \\
\text { tently applied } \\
\text { reference "gold" } \\
\text { standard) } \\
\text { 2. Systematic re- } \\
\text { view }^{2} \text { of Level- } \\
\text { III studies }\end{array}$ & $\begin{array}{r}\text { 1. Limited } \\
\text { alternatives } \\
\text { and costs; } \\
\text { poor esti- } \\
\text { mates } \\
\text { 2. Systemat- } \\
\text { ic review }{ }^{2} \text { of } \\
\text { Level-III } \\
\text { studies }\end{array}$ \\
\hline $\begin{array}{l}\text { Level } \\
\text { IV }\end{array}$ & $\begin{array}{r}\text { Case series (no, } \\
\text { or historical, } \\
\text { control group) }\end{array}$ & Case series & $\begin{array}{r}\text { 1. Case-control } \\
\text { study } \\
\text { 2. Poor reference } \\
\text { standard }\end{array}$ & $\begin{array}{l}\text { No sensitivi- } \\
\text { ty analyses }\end{array}$ \\
\hline $\begin{array}{l}\text { Level } \\
\mathrm{V}\end{array}$ & Expert opinion & $\begin{array}{r}\text { Expert opin- } \\
\text { ion }\end{array}$ & Expert opinion & $\begin{array}{r}\text { Expert opin- } \\
\text { ion }\end{array}$ \\
\hline \multicolumn{5}{|c|}{$\begin{array}{l}\text { 1. All patients were enrolled at the same point in their disease course (incep- } \\
\text { tion cohort) with } \geq 80 \% \text { follow-up of enrolled patients. }\end{array}$} \\
\hline \multicolumn{5}{|c|}{ 2. A study of results from two or more previous studies. } \\
\hline \multicolumn{5}{|c|}{$\begin{array}{l}\text { 3. Patients were compared with a control group of patients treated at the same } \\
\text { time and institution. }\end{array}$} \\
\hline \multicolumn{5}{|c|}{ 4. The study was initiated after treatment was performed. } \\
\hline $\begin{array}{l}\text { 5. Pa } \\
\text { arthr }\end{array}$ & $\begin{array}{l}\text { with a particul } \\
\text { ty) were compa } \\
\text { n, for example, }\end{array}$ & $\begin{array}{l}\text { utcome ("cas } \\
\text { with those w }\end{array}$ & with, for examp & $\begin{array}{l}\text { failed total } \\
\text { ome ("con- }\end{array}$ \\
\hline
\end{tabular}

Downloaded from http://ijssurgery.com/ by guest on April 26, 2023 
quality of evidence from the strength of recommendation or consensus statement.

\section{Results}

Selection of Studies

The search yielded 1,236 potentially relevant citations; of these, 1,057 were excluded based on title and/or abstract. Of the 179 articles that underwent abstract and methods review, 49 papers were extracted for full-text review. Among the final 20 papers which were chosen, two were large database studies, seven were radiographic correlation studies, and 11 were clinical surgical treatment studies (Figure 3). Three of the clinical studies were from the same group and used overlapping patient cohorts ${ }^{6-8}$. Therefore, the most pertinent and applicable data analyses were chosen for inclusion in the appropriate sections of this review. General charactersitics of the 19 papers are listed in Table 3.

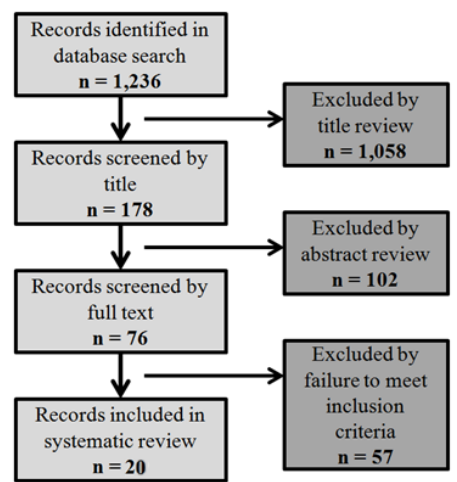

Fig. 3. Literature review flowchart. 
Table 3. Characteristics of included studies.

\begin{tabular}{|c|c|c|c|c|c|c|}
\hline AUTHOR & $\begin{array}{r}\text { Level of evidence (CP judge- } \\
\text { ment on JBJS) }\end{array}$ & $\begin{array}{r}\text { Mean FU } \\
\text { (years) }\end{array}$ & Study Design/Type & N Cases/Grade (G) if available & Patient Factors (previous surgery, age, sex) & Type/Level of Spondylolisthesis \\
\hline 2002_Hanson & $\begin{array}{r}\text { Level IV - therapeutic, non- } \\
\text { comparative retrospective, case } \\
\text { series }\end{array}$ & $\begin{array}{r}4.6 \mathrm{yrs} \\
(2-7)\end{array}$ & Retrospective clinical & $\begin{array}{r}\mathrm{N}=17 \\
(\mathrm{n}=15 \mathrm{G} 4 ; \mathrm{n}=2 \mathrm{G} 3)\end{array}$ & $\begin{array}{r}10 \text { primary and } 7 \text { revision, pediatric and adult } \\
(9-56,20.3 \text { mean }), 15 \mathrm{~F} 2 \mathrm{M}\end{array}$ & Isthmic, L5-S1 \\
\hline 2002_Curylo & $\begin{array}{l}\text { Level III - prognostic, retrospec- } \\
\text { tive study with no control group }\end{array}$ & $\mathrm{n} / \mathrm{a}$ & Retrospective radiographic & $\mathrm{n}=45$ & $\begin{array}{r}\text { Avg age } 22(11-55) \\
37 \mathrm{~F} 16 \mathrm{M}\end{array}$ & Spondyloptosis, bony dysplasia \\
\hline 2002_Hanson & $\begin{array}{r}\text { Level II - prognostic, retrospec- } \\
\text { tive, with control }\end{array}$ & $\mathrm{n} / \mathrm{a}$ & Retrospective radiographic & $\begin{array}{r}\mathrm{N}=80(20 \mathrm{HG}, 20 \mathrm{LG}, 20 \text { adult control, } 20 \text { pe- } \\
\text { diatric control) }\end{array}$ & $\begin{array}{r}\text { Mean age: } \mathrm{HG}(17.7,10-50), \mathrm{LG}(26.6, \\
15-51), \text { adult }(60), \text { ped (11.8) }\end{array}$ & Isthmic \\
\hline 2002_Molinari & $\begin{array}{l}\text { Level III - therapeutic retrospec- } \\
\text { tive comparative study }\end{array}$ & $\begin{array}{r}3.1 \mathrm{yrs} \\
(2-10)\end{array}$ & Retrospective clinical & $\begin{array}{r}\mathrm{N}=31 \text { (Higher Grade tended to get reduction } \\
\text { and internal stabilization) }\end{array}$ & 31 primary, 6 revision, adolescent $(13.5,9-20)$ & Isthmic and dysplastic \\
\hline 2003_Huang & $\begin{array}{l}\text { Level II - prognostic, retrospec- } \\
\text { tive study, with control }\end{array}$ & $\begin{array}{r}\mathrm{n} / \mathrm{a}(\text { preop } \\
\mathrm{x} \text {-rays on- } \\
\text { ly) }\end{array}$ & Retrospective radiographic & $\mathrm{N}=36(\mathrm{n}=22 \mathrm{HG}, \mathrm{n}=14 \mathrm{LG})$ & $\begin{array}{l}\text { Adolescents and adult: Avg } 21.3 \text { yrs (12-53), } \\
24 \text { women, } 12 \text { men, } \mathrm{HG} \text { age } 25.3, \mathrm{LG} \text { age } 15.4\end{array}$ & Isthmic only, L5-S1 only \\
\hline 2005_DeWald & $\begin{array}{l}\text { Level III - prognostic, retrospec- } \\
\text { tive, with no control }\end{array}$ & $\begin{array}{r}6.6 \mathrm{yrs} \\
(1-14)\end{array}$ & $\begin{array}{r}\text { Retrospective review and literature re- } \\
\text { view }\end{array}$ & $\mathrm{N}=21$ & 5 revisions, Avg age 35 yrs (21-68), 13F $8 \mathrm{M}$ & $\begin{array}{r}\text { Developmental (dysplatic) and ac- } \\
\text { quired (isthmic), } \\
\text { L5-S1 and L5-L6 }\end{array}$ \\
\hline 2005_Shufflebarger & $\begin{array}{r}\text { Level IV - therapeutic, case se- } \\
\text { ries }\end{array}$ & $\begin{array}{r}3.3 \mathrm{yrs} \\
(2-5)\end{array}$ & Prospective clinical, single arm cohort & $\mathrm{N}=18$ & Avg age 14 yrs (10-16), 11 women, 7 men & Isthmic dysplastic \\
\hline 2005_Vialle & Level IV - prognostic case series & $\mathrm{n} / \mathrm{a}$ & $\begin{array}{r}\text { Radiographic analysis and clinical re- } \\
\text { view }\end{array}$ & $\mathrm{N}=100$ & Avg age 13.8 yrs (8-17), 67 women, 33 men & $\begin{array}{r}\text { Isthmic (with dysplastic posterior } \\
\text { elements) }\end{array}$ \\
\hline 2006_Helenius & $\begin{array}{l}\text { Level III - therapeutic, retro- } \\
\text { spective comparative study }\end{array}$ & $\begin{array}{r}17.2 \mathrm{yrs} \\
(11-26)\end{array}$ & Retrospective comparative & $\begin{array}{r}\mathrm{n}=21 \text { posterolateral } \\
\mathrm{n}=23 \text { anterior } \\
\mathrm{n}=26 \text { circumferential }\end{array}$ & Avg age 14.4 yrs, 25 women, 45 men & High-grade isthmic \\
\hline \multirow{2}{*}{ 2006_Poussa } & \multirow{2}{*}{$\begin{array}{l}\text { Level III - therapeutic, retro- } \\
\text { spective comparative study }\end{array}$} & \multirow{2}{*}{$\begin{array}{r}14.8 \mathrm{yrs} \\
(11.6-18.7)\end{array}$} & \multirow{2}{*}{$\begin{array}{r}\text { Retrospective follow-up, comparative, } \\
\text { nonrandomized }\end{array}$} & $\mathrm{N}=22, \mathrm{n}=11$ reduction & \multirow{2}{*}{$\begin{array}{r}\text { Avg age } 14.7 \text { yrs (11-18) } 16 \mathrm{~F} 6 \mathrm{M} \text { (split even- } \\
\text { ly between groups) }\end{array}$} & $\begin{array}{r}\text { Isthmic }>60 \%, 3 \text { exaggerated lum- } \\
\text { bar lordosis, } 1 \text { scoliosis }\end{array}$ \\
\hline & & & & $\mathrm{n}=11$ in situ & & $\begin{array}{r}\text { Isthmic }>50 \% \text {, Scoliosis } n=3 \text { due } \\
\text { to slip, Gait problems } n=3\end{array}$ \\
\hline 2006_Vialle & $\begin{array}{r}\text { Level IV - therapeutic, case se- } \\
\text { ries }\end{array}$ & $18 \mathrm{yrs}$ & $\begin{array}{r}\text { Retrospective case series; clinical and } \\
\text { radiographic outcomes }\end{array}$ & $\mathrm{N}=40$, all $\mathrm{HG}$ & $13.5 \mathrm{yrs}(6-18), 25 \mathrm{~F} 15 \mathrm{M}$ & all L5-S1, isthmic and/or dysplas- \\
\hline 2007_Hresko & $\begin{array}{r}\text { Level II - prognostic, retrospec- } \\
\text { tive study }\end{array}$ & $\mathrm{n} / \mathrm{a}$ & $\begin{array}{r}\text { Retrospective review, radiographic } \\
\text { database of HGIS comparison to con- } \\
\text { trols }\end{array}$ & $\begin{array}{r}\mathrm{N}=133 \mathrm{HG} \text {, asymptomatic controls matched by } \\
\text { PI }\end{array}$ & $17 \mathrm{yrs}$ (inclusion $10-40 \mathrm{yrs}$ ), $70 \% \mathrm{~F}$ & $\begin{array}{r}\text { Developmental spondylolisthesis, } \\
\text { L5-S1 }\end{array}$ \\
\hline 2007_Vialle & $\begin{array}{r}\text { Level II - prognostic, retrospec- } \\
\text { tive study }\end{array}$ & $\mathrm{n} / \mathrm{a}$ & $\begin{array}{r}\text { Retrospective radiographic review w/ } \\
\text { healthy control group }\end{array}$ & $\begin{array}{r}\text { Total } \mathrm{N}=244 \text { patients, } \mathrm{N}=300 \text { control; } \mathrm{HG} \\
\mathrm{n}=174: \mathrm{G} 3=98, \mathrm{G} 4=59, \mathrm{G}=17\end{array}$ & $\mathrm{~N}=244: 13.9$ yrs $(6-25), 158 \mathrm{~F} 86 \mathrm{M}$ & Developmental spondylolisthesis \\
\hline $\begin{array}{l}\text { 2008_Rodriguez- } \\
\text { Olaverri }\end{array}$ & $\begin{array}{l}\text { Level III - therapeutic, retro- } \\
\text { spective comparative study }\end{array}$ & $\begin{array}{r}35 \text { months } \\
\text { (24-48) }\end{array}$ & $\begin{array}{l}\text { Retrospective review of two treat- } \\
\text { ments - radiographs and clinical }\end{array}$ & $\begin{array}{r}\mathrm{n}=20 \text { TLIF, } \\
\mathrm{n}=2 \text { Transsacral }\end{array}$ & $30 \mathrm{yrs}(19-48)$ & $\begin{array}{r}\text { Mean grade 3.6 Meyerding } \\
(3-5)\end{array}$ \\
\hline
\end{tabular}




\begin{tabular}{|c|c|c|c|c|c|c|}
\hline 2008_Sasso & $\begin{array}{r}\text { Level IV - prognostic, retrospec- } \\
\text { tive case series }\end{array}$ & $\begin{array}{r}39 \text { months } \\
(30-71)\end{array}$ & Retrospective clinical study & $\mathrm{n}=25$ & $29.8 \mathrm{yrs}$ & $\begin{array}{r}\text { High-grade isthmic spondylolisthe- } \\
\text { sis }\end{array}$ \\
\hline 2009_Hresko & $\begin{array}{r}\text { Level IV - prognostic, retrospec- } \\
\text { tive case series }\end{array}$ & $\begin{array}{r}2-\mathrm{yr} \text { min, } \\
\text { mean } \\
7.5 \pm 5.4\end{array}$ & Retrospective review & $\mathrm{n}=26$ & $13.1+/-3.3$ yrs. $20 \mathrm{~F} 6 \mathrm{M}$ & High-grade spondylolisthesis \\
\hline 2010_Sansur & $\begin{array}{r}\text { Level II - prognostic retrospec- } \\
\text { tive study }\end{array}$ & NA & Retrospective large-scale database & $\mathrm{n}=10,242$ & $\begin{array}{r}\text { Age }>65 \text { has higher comp rate, but not after } \\
\text { adjusting for grade/type }\end{array}$ & $\begin{array}{r}\text { Degenerative and isthmic spondy- } \\
\text { lolisthesis }\end{array}$ \\
\hline \multirow{2}{*}{ 2011_Jalanko } & \multirow{2}{*}{$\begin{array}{l}\text { Level III - therapeutic, retro- } \\
\text { spective comparative study }\end{array}$} & $\begin{array}{r}\text { HG } 18 \text { yrs } \\
(12-25)\end{array}$ & \multirow{2}{*}{ Retrospective comparative study } & $\mathrm{n}=41$ children, $\mathrm{n}=19 \mathrm{HG}$ & 12 yrs (8-14) HG- $13 \mathrm{~F} 6 \mathrm{M}$ & \multirow{2}{*}{$\begin{array}{r}\text { Isthmic spondylolisthesis, low } \\
\text { back pain in most or risk of pro- } \\
\text { gression }\end{array}$} \\
\hline & & $\begin{array}{l}\text { HG } 15 \text { yrs } \\
(11-26)\end{array}$ & & $\begin{array}{l}\mathrm{n}=41 \text { adolescent matched controls (matched for } \\
\text { treatment, gender, and slip severity), } \mathrm{n}=19 \mathrm{HG}\end{array}$ & 16 yrs (13-19) HG- $13 \mathrm{~F} 6 \mathrm{M}$ & \\
\hline \multirow{2}{*}{ 2012_Kasliwal } & \multirow{2}{*}{$\begin{array}{r}\text { Level II - prognostic, retrospec- } \\
\text { tive study }\end{array}$} & \multirow{2}{*}{$\begin{array}{r}\mathrm{n} / \mathrm{a} \text {, short- } \\
\text { term }\end{array}$} & \multirow{2}{*}{ Retrospective study of database } & $\begin{array}{r}\mathrm{N}=165 ; \mathrm{n}=88 \text { pediatric: } \mathrm{n}=40 \mathrm{G} 3, \mathrm{n}=34 \mathrm{G} 4, \\
\mathrm{n}=14 \mathrm{G} 5\end{array}$ & $\begin{array}{r}23 \% \text { revisions (overall). } 13.9 \text { yrs (7-18); } 61 \mathrm{~F} \\
27 \mathrm{M}\end{array}$ & Isthmic in $\mathrm{n}=56$ \\
\hline & & & & $\begin{array}{l}\mathrm{n}=77 \text { adult: } \mathrm{n}=53 \mathrm{G} 3, \mathrm{n}=17 \mathrm{G} 4, \mathrm{n}=7 \mathrm{G} 5 \text {. Isth- } \\
\text { mic } \mathrm{n}=47\end{array}$ & $\begin{array}{r}23 \% \text { revisions (overall). } 44.4 \text { yrs (19-84); } 44 \mathrm{~F} \\
33 \mathrm{M}\end{array}$ & Isthmic in $\mathrm{n}=47$ \\
\hline 2014_Wang & $\begin{array}{r}\text { Level II - prognostic, prospec- } \\
\text { tive cohort }\end{array}$ & N/A & Prospective clinical, two study arms & Isthmic $n=64$ & $44.5 \mathrm{yrs}(13-65) ; 30 \mathrm{~F} 34 \mathrm{M}$ & Isthmic spondylolisthesis on L5 \\
\hline
\end{tabular}




\section{Discussion}

Radiographic Analysis and Risk of Progression Progression of HGS is a major clinical concern for non-surgically treated patients, given the potential for pelvic remodelling and increasing complexity of surgical treatment associated with delayed management. Further, differences arise between pediatric and adult populations, and thus demand different HGS management options.

Seven LOE II studies attempted to identify patients at risk for progression to a higher-grade slip. ${ }^{9-14}$ Five studies reported a correlation between PI and slip grade, ${ }^{9-13}$ all finding that PI was significantly higher in spondylolisthesis patients compared to controls (e.g. $76^{\circ}$ vs. $48.2-53.2^{\circ}$, respectively). ${ }^{11}$ Three studies did not find a difference by grade, ${ }^{9,13}$ but one study was limited by its small population size $(n=22)$ and sole inclusion of patients with a previous fusion. ${ }^{9}$

No specific radiographic parameters were identified as independently associated with risk of progression. There is strong evidence of correlation between PI, age and grade of spondylolisthesis ${ }^{26}$ and between the magnitude of initial slip, slip angle and progression. 9 Wang et al. also elucidated a correlation between spondylolisthesis grade, PT, LL, and exacerbated lower back pain, as measured by the ODI. ${ }^{38}$ Further, the correlation between PI and SS in adults, may explain why patients with high PI values have HGS. Despite these relationships, overall evidence suggests that PI alone is not a reliable predictor of slip progression.

Three LOE II studies and one LOE IV study addressed sacral verticality as measured by SS..$^{12-14}$ Hresko et al. proposed a classification system dividing high grade spondylolisthesis cases into unbalanced and balanced pelvic groups based on the orientation, i.e. radiographic parameters, of the pelvis. Balanced pelvises had higher SS and lower pelvic tilt, whereas unbalanced pelvises incompletely compensate with lower SS and pelvic retroversion, resulting in a higher pelvic tilt (PT) ${ }^{12} \mathrm{~A}$ single retrospective study looking at patients with either unbalanced retroverted or balanced pelvises reported that spondylolisthesis grade, SS, and PT all improved significantly following partial reduction and fusion with instrumentation or casting. ${ }^{15} \mathrm{~A}$ later study by the same group found that SS increases in early grades, then decreases in Grades IV and V, possibly due to loss of contact between L5 and the cranial sacral endplate..$^{13}$ An additional prospective radiographic study concluded a significant negative correlation between SS and severity of low back pain. ${ }^{38}$ Full details on included radiographic studies are available in Table 4. 
Table 4. Summary of radiographic studies.

\begin{tabular}{|c|c|c|c|c|c|c|}
\hline AUTHOR & $\begin{array}{r}\text { Pelvic Incidence } \\
\left({ }^{\circ}\right)\end{array}$ & $\begin{array}{r}\text { Sacral Slope } \\
(\text { SS)/ Inclina- } \\
\text { tion (SI) }\left({ }^{\circ}\right)\end{array}$ & $\begin{array}{r}\text { Slip Angle } \\
\left({ }^{\circ}\right)\end{array}$ & \multicolumn{2}{|c|}{$\begin{array}{r}\text { Additional radiography (findings for radiographic stud- } \\
\text { ies) }\end{array}$} & Conclusion \\
\hline 2002_Curylo & $\begin{array}{r}\text { Control: } 48.2-53.2 \\
\text { Patient: } 76 \pm 10\end{array}$ & NA & NA & & $\begin{array}{r}\text { Sacral Kyphosis }\left(^{\circ}\right): 56 \pm 15 \\
62 \% \text { of patients had } \\
\text { posterior element dysplasia }\end{array}$ & $\begin{array}{l}\text { Progression is linked to shear stress-related to increased verticality of lumbosacral joint, predetermined by } \\
\text { PI and sacral anatomy }\end{array}$ \\
\hline $\begin{array}{l}2002 \text { Han- } \\
\text { son }\end{array}$ & $\begin{array}{r}\text { Ped Control/Adult } \\
\text { Control/LG/HG: } \\
47.4 / 57 / 68.5 / 79\end{array}$ & $\begin{array}{r}\text { SI Ped Con- } \\
\text { trol/Adult } \\
\text { Control/LG/ } \\
\text { HG: } \\
52 / 50 / 48 / 42\end{array}$ & $\begin{array}{r}\text { Ped Control/ } \\
\text { Adult Control/ } \\
\text { LG/HG: }-12 /-15 / \\
9 / 26\end{array}$ & & $\begin{array}{r}\text { Lumbar Lordosis }(\mathrm{LL})\left({ }^{\circ}\right) \\
\text { Ped Control/Adult Control/LG/HG: } \\
58 / 58 / 61 / 58\end{array}$ & $\begin{array}{r}\text { Significant correlation between PI and Meyerding-Newman scores ( } \mathrm{p}=0.03) \text {; PI may be a good predictor of } \\
\text { progression }\end{array}$ \\
\hline \multirow{2}{*}{ 2003_Huang } & HG: $79.6 \pm 1.9$ & SI HG: $34.2 \pm$ & HG: $20.3 \pm 2.8$ & & Slip \%: $79.9 \pm 4.2 \%(p<0.001)$ & \multirow{2}{*}{$\begin{array}{r}\text { No difference in PI or sacral inclination between } \mathrm{HG} \text { and } \mathrm{LG} \text { ( } \mathrm{p}=0.66,0.33 \text {, respectively). Slip percentage } \\
\text { and slip angle are predictive of progression. }\end{array}$} \\
\hline & LG: $72.9 \pm 3.7$ & $\begin{array}{r}\text { SI LG: } 46.5 \pm \\
3.8\end{array}$ & $\begin{aligned} \text { LG: }-8.5 \pm 5.4 \\
\quad(p<0.001)\end{aligned}$ & & Slip \%: $29.5 \pm 3.9 \%(p<0.001)$ & \\
\hline 2005_Vialle & \multicolumn{4}{|c|}{$\begin{array}{r}\text { Analyzed S1 plate for bony hook/outgrowth, condensed and necrotic } \\
\text { anterior edge, round convexity median section. Sagittal inclination, } \\
\text { sacral angle, S1 hypoplasia, }\end{array}$} & $\begin{array}{l}\text { S1 bony hook negatively correlates with lum- } \\
\text { bosacral kyphosis severity; Reducibility is better } \\
\text { without hook/L } 5 \text { bony outgrowth. }\end{array}$ & $\begin{array}{l}\text { S1 index (cranial plate/caudal plate length) correlated with grade slip, lumbosacral kyphosis, and reducibili- } \\
\text { ty of kyphosis }\end{array}$ \\
\hline \multirow{3}{*}{ 2007_Hresko } & $\begin{array}{r}\text { Asymptomatic: } \\
50 \pm 10.7\end{array}$ & $\begin{array}{r}\text { SS Asympto- } \\
\text { matic: } \\
40 \pm 8\end{array}$ & NA & & Asymptomatic: Pelvic tilt (PT) $10 \pm 7.6$ & \multirow{3}{*}{$\begin{array}{l}\text { HG patients have higher PT, SS and PI, but higher PI controls also have high PT and SS. HG divided into } \\
\text { the "unbalanced" high PT/low SS and the "balanced" low PT/high SS. Balanced is more similar to asympto- } \\
\text { matic controls }\end{array}$} \\
\hline & \multirow{2}{*}{$78.9 \pm 12.1$} & $\begin{array}{r}\text { SS Balanced: } \\
59.9 \pm 11.2\end{array}$ & NA & & $\begin{array}{r}\text { Balanced: Pelvic tilt }(\mathrm{PT}) 21.3 \pm 8.2 \\
\text { Slip \%: } 68 \pm 46.9 \%(\mathrm{p}=0.13)\end{array}$ & \\
\hline & & $\begin{array}{l}\text { SS Unbal- } \\
\text { anced: } \\
40.3 \pm 9.0\end{array}$ & NA & & $\begin{array}{r}\text { Unbalanced: PT } 36.5 \pm 8.0 \\
\text { Slip \%: } 78 \pm 23.7 \%(\mathrm{p}=0.13)\end{array}$ & \\
\hline \multirow{2}{*}{ 2007_Vialle } & $\begin{array}{r}\text { Control: } \\
54.7 \pm 10.7^{*}\end{array}$ & $\begin{array}{l}\text { SS Control: } \\
41.9 \pm 8.4^{*}\end{array}$ & NA & & $\begin{array}{r}\text { LL }\left({ }^{\circ}\right):-43.13 \pm 11.2^{*} \\
\mathrm{PT}\left({ }^{\circ}\right): 13.2 \pm 6.1^{*} \\
\text { Lumbosacral angle }(\mathrm{LSA})\left({ }^{\circ}\right): 109.94 \pm 7.2^{*}\end{array}$ & \multirow{2}{*}{$\begin{array}{l}\text { PI is significantly higher in spondylolisthesis, but not clearly correlated with the grade of slipping; lum- } \\
\text { bosacral kyphosis evaluated by LSA possibly the most important factor. }\end{array}$} \\
\hline & $\begin{array}{l}\text { Patient: } \\
73.1 \pm 11.3^{*}\end{array}$ & $\begin{array}{r}\text { SS Patient: } \\
46.6 \pm 12.6^{*}\end{array}$ & NA & & $\begin{array}{l}\text { LL }\left(^{\circ}\right):-70.22 \pm 17.3^{*} \\
\operatorname{PT}\left({ }^{\circ}\right): 26.5 \pm 10.9^{*} \\
\text { LSA }\left(^{\circ}\right): 82.3 \pm 21.2^{*}\end{array}$ & \\
\hline 2014_Wang & $\begin{array}{r}\text { ODI } 0-22: \\
74.6 \pm 11.6\end{array}$ & $\begin{array}{r}\text { ODI 0-22: } \\
52.3 \pm 9.4^{*}\end{array}$ & NA & & $\begin{array}{r}\text { ODI 0-22: } \\
\text { Spondylolisthesis grade: } 62.8 \pm 10.4^{*} \\
\text { LL }\left({ }^{\circ}\right): 56.5 \pm 9.4^{*} \\
\operatorname{PT}\left({ }^{\circ}\right): 22.3 \pm 6.3^{*} \\
\operatorname{TK}\left({ }^{\circ}\right): 41.2 \pm 8.8 \\
\text { SFHD }\left(^{\circ}\right): 44.9 \pm 10.3^{*} \\
\text { SFVD }\left(^{\circ}\right): 109.5 \pm 15.6^{*} \\
\text { SC7D }\left(^{\circ}\right): 23.2 \pm 34.3^{*}\end{array}$ & $\begin{array}{l}\text { Spondylolisthesis grade, SS, PT, SC7D, LL, SFVD, SFHD, PT/SS, SFHD/SFVD, LL/TK are significantly } \\
\text { associated with clinical symptoms of severe isthmic spondylolisthesis; SS (-0.981, strong) and SFVD } \\
\text { (-0.802, strong) are most significant correlations with low back pain }\end{array}$ \\
\hline
\end{tabular}




\begin{tabular}{|c|c|c|}
\hline $\begin{array}{l}\text { ODI 23-45: } \\
77.9 \pm 12.7\end{array}$ & $\begin{array}{c}\text { ODI } 23-45 \\
44.5 \pm 9.0 * \\
{ }^{*} p<0.0001\end{array}$ & $\begin{array}{r}\text { ODI 23-45: } \\
\text { Spondylolisthesis grade: } 74.5 \pm 11.5^{*} \\
\operatorname{LL}\left(^{\circ}\right): 56.5 \pm 9.4^{*} \\
\operatorname{PT}\left({ }^{\circ}\right): 33.4 \pm 8.1^{*} \\
\operatorname{TK}\left({ }^{\circ}\right): 37.6 \pm 7.4 \\
\text { SFHD }\left(^{\circ}\right): 53.5 \pm 10.8^{*} \\
\text { SFVD }\left({ }^{\circ}\right): 81.1 \pm 11.7^{*} \\
\text { SC7D }\left({ }^{\circ}\right): 41.1 \pm 37.4^{*}\end{array}$ \\
\hline
\end{tabular}

Downloaded from http://ijssurgery.com/ by guest on April 26, 2023 
There is good and increasing evidence in the literature that surgical treatment of HGS be based on pelvic parameters. Hresko et al. noted that increased PI with HGS is matched by specific changes in SS and PT that separates patients based on balance, with reference to an asymptomatic control group: 'balanced' HGS patients were similar to asymptomatic patients (lower PT, higher SS angles), while 'unbalanced' HGS with higher PT and lower SS were more characteristic of symptomatic patients. Hresko et al. also established a classification system for categorizing lumboscaral spondylolisthesis into low vs. high slip grade. Low slip grades are further categorized into low $\left(<45^{\circ}\right)$, normal $\left(45-60^{\circ}\right)$ or high PI $\left(\geq 60^{\circ}\right)$ and the high slip grades into balanced (high SS/low PT) or unbalanced (low SS/high PT). ${ }^{28}$ These findings may be related to the complex relationships and adjustment mechanisms between pelvis and global alignment. ${ }^{29}$ Lafage et al. suggest using a morphologic pelvic parameter, namely PI, and spinal parameters modifiable through surgery, such as lumbar lordosis (LL) or thoracic kyphosis (TK), to predict postoperative sagittal alignment. ${ }^{30}$

Furthermore, determining risk of progression based on radiographic parameters is complicated by the anatomical changes characteristic of HGS. S1 remodeling by dysplastic L5 and loss of L4 and S1 articulation makes border identification dificult. Vialle et al. noted that patients with low SS had a more easily reduceable spondylolisthesis ${ }^{14}$ and that SS, PT, and LL measurements in HGS were compensatory for high PI. ${ }^{13}$ Schuller et al found that BMI was significantly higher in a spondylolisthesis group compared to control and suggested that increased PT in HGS patients most likely results from a compensatory posterior pelvic tilt. ${ }^{31}$ The association between changes of pelvic parameters, BMI, and HGS may be related to the increasing gravitational forces seen at the lumbosacral junction associated with an increasing SS, possibly making the translational and angular components of the slip more difficult to reduce.

Surgical Approach and Instrumentation

Four LOE III and five LOE IV studies with detailed operative descriptions ${ }^{7,16-23}$ and two LOE II database studies addressing surgical approach were included. ${ }^{24,25}$ Table 5 and Table 6 report the data extracted concerning surgical approaches and surgical outcomes, respectively. Surgical approaches used to treat HGS included anterior lumbar interbody fusion (ALIF), posterior/posterolateral only (PLF), transforaminal lumbar interbody fusion (TLIF) \pm PLF, posterior lumbar interbody fusion (PLIF) \pm PLF, and circumferential fusion. Reported outcome measures varied between authors, which include fusion status, neurologic and/or clinical deficit, patients' perception of outcomes measured with post-operative questionanaires, and complications. Three LOE III studies directly compared circumferential fusions with interbody support to PLF-only procedures. ${ }^{7,17,20} \mathrm{~Pa}$ tients treated with circumferential fusion either had better long-term results, ${ }^{7,17}$ or the techniques were similar in outcome..$^{20}$ One LOE III study included an anterior-only cohort ${ }^{7}$ and found that anterior-only patients scored better in all SRS scores compared to PLF-only, but worse than circumferential group. This study found that overall the circumferential fusion approach resulted in significantly improved clinical, radiographic, and SRS scores post-operatively, compared to posterolateral or anterior fusions. ${ }^{11} \mathrm{~A}$ LOE IV study reported no difference in clinical and radiographic outcomes and complications between PLIF and ALIF. ${ }^{21}$ TLIF, ALIF, and PLIF were studied in the included articles, however none of the considered studies directly compared the three procedures. ${ }^{7,16,17,19,21-23}$ 
Table 5. Summary of Surgical Strategy

\begin{tabular}{|c|c|c|c|c|c|}
\hline AUTHOR & Approach & $\begin{array}{r}\text { Fusion levels/ Instrumenta- } \\
\text { tion }\end{array}$ & Graft & Decompression & Reduction \\
\hline 2002_Hanson & $\begin{array}{r}\text { Posterior: } \mathrm{n}=2 \\
\text { Ant/Post: } \mathrm{n}=15 \text { (performed in } \\
\text { two separate stages, usu. } 5 \\
\text { days later) }\end{array}$ & $\begin{array}{r}\mathrm{n}=2 \text { none, } \mathrm{n}=11 \text { L4-S1, } \mathrm{n}=1 \\
\text { L3-S1+ilium, } \mathrm{n}=1 \\
\text { L4-S1+ilium, } \mathrm{n}=1 \\
\text { L5-S1+ilium, } \mathrm{n}=1 \\
\text { T5-sacrum+ilium; Pedicle } \\
\text { screws. }\end{array}$ & $\begin{array}{r}\text { Fibular dowel graft (allograft } \\
n=11 \text {, autograft } n=6 \text { ) }\end{array}$ & $\begin{array}{r}\text { Wide decom- } \\
\text { pression of the } \\
\text { L5-S1 root }\end{array}$ & $\begin{array}{r}n=15: \text { Grade } 4 / 3 \text { Pre-Op to } \\
\text { Grade } 1 / 3 \text { Post-Op } \\
n=2: \text { no change }\end{array}$ \\
\hline \multirow{3}{*}{ 2002_Molinari } & $\begin{array}{l}\mathrm{n}=18 \text { Posterior; } \\
\mathrm{n}=11 \text { Group } 1 \mathrm{~A}\end{array}$ & $\begin{array}{r}\text { Group } 1 \mathrm{~A}=\mathrm{L} 4 \text {-sacrum } \\
\text { No instrumentation }\end{array}$ & NA & $\begin{array}{r}\text { Group } 1 \mathrm{~A}=\mathrm{No} \\
\text { decompression }\end{array}$ & Group $1 \mathrm{~A}=$ in situ \\
\hline & $\mathrm{n}=7$ Group 1B & $\begin{array}{r}\text { Group 1B=L4-sacrum Posteri- } \\
\text { or instrumentation }\end{array}$ & NA & $\begin{array}{r}\text { Group } 1 \mathrm{~B}=\text { Pos- } \\
\text { terior decom- } \\
\text { pression }\end{array}$ & Group $1 \mathrm{~B}=6 / 7$ patients \\
\hline & $\begin{array}{l}\mathrm{n}=19 \text { Circumferential with an- } \\
\text { terior support (Group 2) } \\
\mathrm{n}=16 \text { separate anterior ap- } \\
\text { proach, } \mathrm{n}=3 \text { PLIF in lieu of } \\
\text { anterior approach }\end{array}$ & $\begin{array}{r}\text { Circumferential with anterior } \\
\text { structural support; } \mathrm{n}=3 \text { Struc- } \\
\text { tural graft and posterior fixa- } \\
\text { tion }\end{array}$ & Anterior structural graft & $\begin{array}{r}\text { Group 2=Poste- } \\
\text { rior decompres- } \\
\text { sion }\end{array}$ & Group $2=19 / 19$ patients \\
\hline 2005_DeWald & $\begin{array}{r}\mathrm{n}=10, \text { Ant. interbody cage/ } \\
\text { graft } \mathrm{n}=9, \text { Post. interbody } \\
\text { cage/graft } \mathrm{n}=2, \text { Ant. Pedicle } \\
\text { screws/graft }\end{array}$ & $\begin{array}{r}\mathrm{n}=1 \mathrm{NA}, \mathrm{n}=11 \text { L4-S1, } \\
\mathrm{n}=5 \mathrm{~L} 5-\mathrm{S} 1, \mathrm{n}=3 \mathrm{~L} 3-\mathrm{S} 1, \mathrm{n}=1 \\
\mathrm{~L} 4-\mathrm{L} 6 \\
\text { Interbody cages/strut graft; } \\
\text { Pedicle screws }\end{array}$ & $\begin{array}{l}\text { Bone on bone, fibermetal } \\
\text { trans-sacral strut, fibular allo- } \\
\text { graft trans-sacral strut, auto- } \\
\text { graph cortical iliac trans-sacral } \\
\text { strut }\end{array}$ & NA & $\begin{array}{r}n=16 / 21 \text { (14 partial reduc- } \\
\text { tion })\end{array}$ \\
\hline 2005_Shufflebarger & $\begin{array}{r}\text { Posterior lumbar interbody fu- } \\
\text { sion }\end{array}$ & $\begin{array}{r}\mathrm{n}=18 \mathrm{~L} 5-\mathrm{S} 1 \text {; Harm's cage, pos- } \\
\text { terior monosegmental com- } \\
\text { pression instrumentation with } \\
\text { pedicular fixation }\end{array}$ & Autogenous iliac crest graft & $\begin{array}{r}\text { Gill decompres- } \\
\text { sion }\end{array}$ & $\begin{array}{r}\mathrm{n}=18(\% \text { slip avg pre-op } \\
77 \% \text {->post-op } 13 \%)\end{array}$ \\
\hline \multirow{2}{*}{ 2006_Poussa } & Anterior/ Posterolateral & $\begin{array}{r}\mathrm{L} 4-\mathrm{S} 1 \mathrm{n}=9, \mathrm{~L} 5-\mathrm{S} 1 \mathrm{n}=2, \\
\text { transpedicular magerl/dick, an- } \\
\text { terior L5-S1 }\end{array}$ & NA & $\begin{array}{r}\text { Laminectomy } \\
\mathrm{n}=11 \text { (all) }\end{array}$ & Yes \\
\hline & $\begin{array}{l}\text { Anterior/ Posterolateral with- } \\
\text { out instrumentation }\end{array}$ & $\begin{array}{l}\mathrm{L} 4-\mathrm{S} 1 \mathrm{n}=7, \mathrm{~L} 5-\mathrm{S} 1 \mathrm{n}=4 \text {, no in- } \\
\text { strumentation, anterior L5-S1 }\end{array}$ & NA & $\begin{array}{r}\text { Laminectomy } \\
\mathrm{n}=7\end{array}$ & $\begin{array}{r}\text { NO (some due to interbody } \\
\text { grafts) }\end{array}$ \\
\hline \multirow{3}{*}{ 2006_Helenius } & $\begin{array}{r}\text { Group 1A: Posterolateral in } \\
\text { situ }\end{array}$ & $\begin{array}{r}\mathrm{n}=4 \text { L5-sacrum, } \mathrm{n}=16 \\
\text { L4-sacrum, } \mathrm{n}=1 \mathrm{~L} 3 \text {-sacrum; } \\
\text { No instrumentation }\end{array}$ & $\begin{array}{r}\text { Autologous iliac crest bone } \\
\text { graft }\end{array}$ & \multirow{3}{*}{ Not performed } & None \\
\hline & $\begin{array}{l}\text { Group 1B: Ant intercorporeal } \\
\text { fusion, transperitoneal, resect- } \\
\text { ed ant/inf edge L5 for access } \\
\text { if necessary }\end{array}$ & $\begin{array}{r}\mathrm{n}=23 \text { L5-S1 } \\
\text { No instrumentation }\end{array}$ & $\begin{array}{l}\text { Autologous iliac crest bone } \\
\text { graft; wedge interbody grafts }\end{array}$ & & $\begin{array}{r}\text { From patient's positioning on } \\
\text { operating table and wedge- } \\
\text { shaped grafts }\end{array}$ \\
\hline & $\begin{array}{l}\text { Group 2: Circumferential: an- } \\
\text { terior/posterolateral, } \\
\mathrm{n}=17 \text { one operation, } \mathrm{n}=9 \\
\text { staged }\end{array}$ & $\begin{array}{r}\mathrm{n}=14 \text { L4-S1, } \mathrm{n}=12 \text { L5-S1 No } \\
\text { instrumentation }\end{array}$ & Autogenous bone graft & & None \\
\hline 2006_Vialle & $\begin{array}{r}\text { Same-day, staged posterior- } \\
\text { anterior approach }\end{array}$ & $\begin{array}{r}\text { Anterior plate to } S 1 \text {, plates } \\
\text { through } S 1 \text { to iliac wings } n=40 \\
\text { Instrumentation in all patients }\end{array}$ & $\begin{array}{r}\text { Posterior: No grafting Anteri- } \\
\text { or: ICBG through anterior } \\
\text { plate }\end{array}$ & $\begin{array}{r}\text { Posterior de- } \\
\text { compression } \\
\text { and removal of } \\
\text { posterior arch of } \\
\text { L5 }\end{array}$ & $\begin{array}{r}\text { Complete reduction in all pa- } \\
\text { tients }\end{array}$ \\
\hline $\begin{array}{l}\text { 2008_Rodriguez- } \\
\text { Olaverri }\end{array}$ & $\begin{array}{l}\text { Group A: Unilateral TLIF } \\
\text { Posterior with transforaminal } \\
\text { lumbar interbody implants }\end{array}$ & $\begin{array}{r}\text { L4, L5, S1, ilium } \\
\text { Rods, screws and two titanium } \\
\text { mesh cages }\end{array}$ & $\begin{array}{r}\text { Posterolateral: autogenous lo- } \\
\text { cal graft + allograft }\end{array}$ & $\begin{array}{r}\text { L5 nerve root, } \\
\text { L5 arch and } \\
\text { pars interarticu- } \\
\text { laris decompres- } \\
\text { sion }\end{array}$ & $\begin{array}{l}\text { Partial reduction via inter- } \\
\text { body packed titanium cage } \\
\text { and hip extension positioning }\end{array}$ \\
\hline
\end{tabular}

Downloaded from http://ijssurgery.com/ by guest on April 26, 2023 


\begin{tabular}{|c|c|c|c|c|c|}
\hline & $\begin{array}{l}\text { Group B: Transsacral Posteri- } \\
\text { or only }\end{array}$ & $\begin{array}{r}\text { Transsacral screws thru L5-S1, } \\
\text { pedicle screws L4 }\end{array}$ & $\begin{array}{r}\text { Posterolateral: autogenous lo- } \\
\text { cal graft + allograft }\end{array}$ & $\begin{array}{r}\text { L5 nerve root, } \\
\text { L5 arch and } \\
\text { pars interarticu- } \\
\text { laris decompres- } \\
\text { sion }\end{array}$ & $\begin{array}{r}\text { Partial reduction via hip ex- } \\
\text { tension positioning }\end{array}$ \\
\hline 2008_Sasso & $\begin{array}{r}\mathrm{n}=8 \text { Posterior-only, } \mathrm{n}=17 \\
\text { Anterior-posterior (paramedi- } \\
\text { an rectus-sparing mini-open } \\
\text { retroperitoneal) same-day } \\
\text { surgery }\end{array}$ & L4-S1 Pedicle screws & $\begin{array}{l}\text { Posterior: Fibular allograft } \\
\text { Anterior: Fibular strut graft } \\
\text { plus femoral ring allograft }\end{array}$ & $\begin{array}{r}\text { S1 Gill laminec- } \\
\text { tomy }\end{array}$ & $\begin{array}{r}\text { Reduced with patient posi- } \\
\text { tioning, no additional at- } \\
\text { tempts at reduction }\end{array}$ \\
\hline 2009_Hresko & $\begin{array}{r}\mathrm{n}=21 \text { Posterolateral, } \mathrm{n}=5 \text { Pos- } \\
\text { terolateral }+ \text { anterior discecto- } \\
\text { my/interbody fusion }\end{array}$ & $\begin{array}{r}\text { L4-sacrum } \\
\mathrm{n}=13 \text { Jackson intrasacral rods, } \\
\mathrm{n}=13 \text { Luque box instrumenta- } \\
\text { tion }\end{array}$ & NA & $\begin{array}{r}\text { Laminectomy } \\
\text { and nerve root } \\
\text { decompression } \\
\text { on case-by-case } \\
\text { basis }\end{array}$ & $\begin{array}{r}\text { Partial reduction achieved by } \\
\text { prone positioning and lumbar } \\
\text { lordosis. Achieved } 10^{\circ} \text { reduc- } \\
\text { tion in } 10 \% \text { of all patients }\end{array}$ \\
\hline
\end{tabular}

Downloaded from http://ijssurgery.com/ by guest on April 26, 2023 
Table 6. Surgical Outcome, Complications and Clinical Recommendations Per Author.

\begin{tabular}{|c|c|c|c|c|c|c|}
\hline AUTHOR & Approach/Study Groups & $\begin{array}{r}\text { Radiography, fu- } \\
\text { sion status } \\
\text { postop }\end{array}$ & Neuro/clinical deficit & $\begin{array}{r}\text { Change in clini- } \\
\text { cal evaluation } \\
\text { (e.g. ODI, SF-12, } \\
\text { others) }\end{array}$ & Complications & Clinical Recommendations \\
\hline 2002_Hanson & $\begin{array}{r}\text { Posterior: } \mathrm{n}=2 \\
\text { Ant/Post: } \mathrm{n}=15\end{array}$ & $\begin{array}{r}\text { Posterior: Grade } 1 \\
(\mathrm{n}=15) \text {, grade } 2 \\
(\mathrm{n}=1), \text { no fusion } \\
(\mathrm{n}=1) \text { Anterior: } \\
\text { Grade } 1(\mathrm{n}=16), \\
\text { broken strut } \\
(\mathrm{n}=1) .\end{array}$ & NA & $\begin{array}{r}\text { Post-op ODI= } \\
11.4, \\
\text { SRS }=37.3, \\
\text { SRS satisfaction= } \\
14.1\end{array}$ & $\begin{array}{r}1 \text { broken strut graft (in situ anterior } \\
\text { only), no neurological deficits, no } \\
\text { infection }\end{array}$ & $\begin{array}{l}\text { Partial reduction of high-grade isthmic spondy- } \\
\text { lolisthesis with fibular strut grafting is a safe, ef- } \\
\text { fective procedure. No difference between allograft } \\
\text { and autograft }\end{array}$ \\
\hline \multirow{3}{*}{ 2002_Molinari } & Group 1A $(n=11)$ & $\begin{array}{r}\text { Group } 1 \mathrm{~A}=45 \% \\
\text { pseudarthrosis }\end{array}$ & \multirow{3}{*}{$\begin{array}{l}\text { No neurologic deficit in patients treated with in } \\
\text { situ; Transient neurologic deficits }(\mathrm{n}=4 / 26) \text {; Failed } \\
\text { intra-op wake-up test }(\mathrm{n}=1) \text { that resolved immedi- } \\
\text { ately after releasing the reduction, foot drops ( } \mathrm{n}=2) \\
\text { and bilateral extensor hallucis longus weakness } \\
\qquad(\mathrm{n}=1)\end{array}$} & $\begin{array}{r}\text { Pain score: } 3 / 10 \\
\text { Function score: } \\
\mathrm{NA} \\
\text { Satisfaction score: } \\
8.4 / 11\end{array}$ & $36 \%$ had progression & \multirow{3}{*}{$\begin{array}{l}\text { Anterior structural grafting combined with posteri } \\
\text { or instrumentation and fusion is effective i } \\
\text { achieving fusion in HGS patients. Outcomes fo } \\
\text { function, pain, and satisfaction are excellent i } \\
\text { those patients who obtain solid fusion regardles } \\
\text { of the surgical procedur }\end{array}$} \\
\hline & Group $1 \mathrm{~B}(\mathrm{n}=7)$ & $\begin{array}{r}\text { Group } 1 \mathrm{~B}=29 \% \\
\text { pseudarthrosis }\end{array}$ & & $\begin{array}{r}\text { Pain score: } 3.6 / 10 \\
\text { Function score: } \\
12.6 / 15 \\
\text { Satisfaction score: } \\
9.4 / 11\end{array}$ & $\begin{array}{l}29 \% \text { instrumentation failure and par- } \\
\text { tial loss of reduction }\end{array}$ & \\
\hline & Group 2: Circumferential $(\mathrm{n}=19)$ & $\begin{array}{l}\text { Group } 2=0 \% \\
\text { pseudarthrosis }\end{array}$ & & $\begin{array}{r}\text { Pain score: } 2.5 / 10 \\
\text { Function score: } \\
13.7 / 15 \text { Satisfac- } \\
\text { tion score: } 9.7 / 11\end{array}$ & $\begin{array}{l}11 \% \text { implant complications and par- } \\
\text { tial loss of reduction }\end{array}$ & \\
\hline 2005_DeWald & $\begin{array}{r}\text { Ant. interbody cage/graft }(\mathrm{n}=10) \text {, } \\
\text { post. interbody cage/graft }(\mathrm{n}=9), \\
\text { anterior pedicle screws/graft }(\mathrm{n}=2)\end{array}$ & $0 \%$ pseudarthrosis & $\begin{array}{l}\text { Hypesthesias or dysesthesias on the dorsum of one } \\
\text { or both feet }(\mathrm{n}=8) \text {; extensor hallus longus weak- } \\
\text { ness }(\mathrm{n}=2) \text {; cauda equina syndrome }(\mathrm{n}=1)\end{array}$ & $\begin{array}{r}\text { Used an unofficial } \\
\text { clinical evaluation } \\
\text { format: } 12 \text { excel- } \\
\text { lent, } 7 \text { good, } 1 \\
\text { fair, and } 1 \text { poor } \\
\text { clinical outcome }\end{array}$ & $\begin{array}{r}\text { Instrumentation failure }(\mathrm{n}=1) \text {, Iliac } \\
\text { vein thrombosis, pulmonary embo- } \\
\text { lus, pancreatitis, and temporary ret- } \\
\text { rograde ejaculation ( } \mathrm{n}=1 \text { each) }\end{array}$ & $\begin{array}{l}\text { Pediatric patients can be treated successfully by } \\
\text { non-instrumented posterior in situ fusion, but in- } \\
\text { strumentation should be used in adults. Recom- } \\
\text { mends use of adjunctive fixation for adults. }\end{array}$ \\
\hline 2005_Shufflebarger & Posterior lumbar interbody fusion & $\begin{array}{r}\text { All patients } \\
\text { achieved } \\
\text { arthrodesis }\end{array}$ & No neurologic complications & NA & $\begin{array}{r}\text { Urinary tract infections }(\mathrm{n}=3) \text {, ileus } \\
(\mathrm{n}=2) \text {, cholelithiasis }(\mathrm{n}=1), \text { no infec- } \\
\text { tious or instrumentation complica- } \\
\text { tions }\end{array}$ & $\begin{array}{l}\text { Reducing slip and lumbosacral kyphosis will pro- } \\
\text { vide ideal biomechanical environment. Structural } \\
\text { anterior column support and posterior transpedicu- } \\
\text { lar instrumentation provide more resistance to } \\
\text { shear forces than posterior instrumentation alone. }\end{array}$ \\
\hline 2006_Helenius & Posterolateral in situ & $\begin{array}{r}\text { Nonunion } \\
(\mathrm{n}=3) \text { Progression } \\
(\mathrm{n}=4)\end{array}$ & $\begin{array}{r}\text { General back pain }(\mathrm{BP})(\mathrm{n}=9) \mathrm{BP} \text { radiating down } \\
\text { leg }(\mathrm{n}=5) \text { Neuro deficiencies/ hamstring tightness } \\
\qquad(\mathrm{n}=14)\end{array}$ & $\begin{array}{r}\text { SRS }=89.7 . \text { ODI } \\
=9.7: \text { severely } \\
\text { disabled }(\mathrm{n}=1), \\
\text { moderately dis- } \\
\text { abled }(\mathrm{n}=2) \mathrm{VAS}= \\
22.6\end{array}$ & Nonunion $(\mathrm{n}=3)$ Re-operation $(\mathrm{n}=1)$ & $\begin{array}{l}\text { Circumferential fusion provided significantly bet- } \\
\text { ter long-term clinical, radiographic, and SRS total } \\
\text { score than posterolateral or anterior fusion for } \\
\text { HGIS }\end{array}$ \\
\hline
\end{tabular}




\begin{tabular}{|c|c|c|c|c|c|c|}
\hline & $\begin{array}{l}\text { Anterior intercorporeal fusion, } \\
\text { trans-peritoneal }\end{array}$ & Progression $(\mathrm{n}=1)$ & & $\begin{array}{r}\mathrm{SRS}=93.2, \mathrm{ODI} \\
=8.9: \text { moderately } \\
\text { disabled }(\mathrm{n}=4), \\
\mathrm{VAS}=24.1 ; \\
\text { scored best on } \\
\text { SRS self-image }\end{array}$ & $\begin{array}{r}\text { Post-op peroneal palsy }(\mathrm{n}=1), \mathrm{L} 5 / \mathrm{S} 1 \\
\text { radicular pain }(\mathrm{n}=3), \text { spondyloptosis } \\
\qquad(\mathrm{n}=1)\end{array}$ & \\
\hline & Circumferential & $\begin{array}{r}\text { Nonunion }(\mathrm{n}=1) \\
\text { Progression }(\mathrm{n}=3) \\
\text { Least lumbosacral } \\
\text { kyphosis progres- } \\
\text { sion }\end{array}$ & & $\begin{array}{r}\mathrm{SRS}=100, \mathrm{ODI}= \\
3.0, \mathrm{VAS}=5.5 . \\
\text { Scored best on } \\
\text { SRS pain and } \\
\text { function }\end{array}$ & $\begin{array}{r}\text { Nonunion }(\mathrm{n}=1) \text {, wound infection } \\
(\mathrm{n}=1) \text {, scoliosis and gait difficulties } \\
\qquad(\mathrm{n}=1)\end{array}$ & \\
\hline \multirow{2}{*}{ 2006_Poussa } & Reduction $(\mathrm{n}=11)$ & $\begin{array}{r}18 \% \text { pseudarthro- } \\
\text { sis }(\mathrm{n}=2)\end{array}$ & L5 nerve root injury during decompression $(\mathrm{n}=1)$ & $\begin{array}{r}\mathrm{ODI}=7.2, \mathrm{SRS}= \\
90\end{array}$ & $\begin{array}{r}\text { Mild muscle atrophy: iliopsoas } \\
(\mathrm{n}=7) \text {, back L3 }(\mathrm{n}=4) / \mathrm{L} 5(\mathrm{n}=6) \text {; Se- } \\
\text { vere atrophy: back L5 }(\mathrm{n}=3)\end{array}$ & \multirow{2}{*}{$\begin{array}{l}\text { Fusion in situ should be considered as a method of } \\
\text { choice in severe L } 5 \text { isthmic spondylolisthesis }\end{array}$} \\
\hline & In situ $(\mathrm{n}=11)$ & $\begin{array}{l}\text { All fusions healed } \\
\text { appropriately }\end{array}$ & Immediate post-op peroneal palsy $18 \%(\mathrm{n}=2)$ & $\begin{array}{r}\text { ODI }=1.6, \text { SRS }= \\
103.9, \text { better SRS } \\
\text { pain and post-op } \\
\text { function }\end{array}$ & $\begin{array}{r}\text { Mild muscle atrophy: iliopsoas } \\
(\mathrm{n}=2) \text {, back } \mathrm{L} 3(\mathrm{n}=2) / \mathrm{L} 5(\mathrm{n}=4) \mathrm{Se}- \\
\text { vere atrophy: iliopsoas }(\mathrm{n}=1)\end{array}$ & \\
\hline 2006_Vialle & $\begin{array}{r}\text { Same-day, staged posterior-anterior } \\
\text { approach }\end{array}$ & $\begin{array}{r}\text { Fusion achieved } \\
\text { in all patients }\end{array}$ & $\begin{array}{r}\mathrm{BP}(\mathrm{n}=12), \mathrm{BP} / \mathrm{bilateral} \text { leg pain }(\mathrm{n}=11), \mathrm{BP} / \text { unilat- } \\
\text { eral leg pain }(\mathrm{n}=9) \text {, radicular }(\mathrm{n}=6) \text {, neuro compli- } \\
\text { cations }(\mathrm{n}=12) \text { : } \mathrm{L} 5 \text { incomplete deficit }(\mathrm{n}=5) \text {, unilat- } \\
\text { eral } \mathrm{L} \text { incomplete deficit }(\mathrm{n}=6) \text {, unilateral L5 hy- } \\
\text { poesthesia }(\mathrm{n}=1) ; 10 / 12 \text { recovered neurologically } \\
\text { within } 18 \text { months. }\end{array}$ & $\begin{array}{r}\text { Beaujon function- } \\
\text { al score: Pre-op } \\
\text { mean } 14.2(5-20) ; \\
\text { Post-op } 20+\text { in } \\
\mathrm{n}=35 ; 15-19 \text { in } \\
\mathrm{n}=5 \text { from BP/leg } \\
\text { pain. } \\
\text { JOA Pre-op mean } \\
10(2-15) ; \text { Post-op } \\
15 \text { in } \mathrm{n}=35 ; 12-14 \\
\text { for } \mathrm{n}=5 .\end{array}$ & $\begin{array}{r}\text { Intra-op complications: iliac vein le- } \\
\text { sion and technical difficulty with } \\
\text { plate }(\mathrm{n}=2) . \\
\text { Implant complications: Kyphosis } \\
\text { due to plate hitting L4-5 (n=2), bro- } \\
\text { ken screws (n=2), late infections } \\
(\mathrm{n}=5)\end{array}$ & $\begin{array}{l}\text { This technique proved to provide an excellent fu- } \\
\text { sion rate and satisfactory final functional out- } \\
\text { comes, but due to the unusually high rate of late } \\
\text { infections that may be related to the surgical ap- } \\
\text { proach, we do not recommend this technique for } \\
\text { HGIS treatment. }\end{array}$ \\
\hline \multirow{2}{*}{$\begin{array}{l}\text { 2008_Rodriguez- } \\
\text { Olaverri }\end{array}$} & $\begin{array}{r}\text { Group A: Unilateral TLIF Posterior } \\
\text { with transforaminal lumbar inter- } \\
\text { body implants }\end{array}$ & $100 \%$ fusion & $\begin{array}{r}\text { Back/leg pain resolved in 90\%, no neurologic } \\
\text { deficits }\end{array}$ & $\begin{array}{r}\text { SRS pre-op: pain } \\
4.8, \\
\text { self-image 3.8, fxn } \\
4.8 ; \text { SRS pos-opt: } \\
\text { pain 4.6, self- } \\
\text { image 3.6, fxn } 4.5\end{array}$ & Durotomy $(\mathrm{n}=7)$, infections $(\mathrm{n}=3$ ) & \multirow{2}{*}{$\begin{array}{l}\text { Both procedure A and B } \\
\text { appear to be safe and effective surgically and radi- } \\
\text { ographically, but we must note that the average } \\
\text { operation time was longer in procedure A ( } 4.45 \\
\text { hrs) than in procedure B ( } 3.25 \mathrm{hrs})\end{array}$} \\
\hline & $\begin{array}{l}\text { Group B: Transsacral Posterior on- } \\
\text { ly }\end{array}$ & $\begin{array}{r}\text { Solid fusion in } 19 / \\
20 \text { subjects }\end{array}$ & $\begin{array}{r}\text { Back/leg pain resolved in } 80 \% \text {, no neurologic } \\
\text { deficits }\end{array}$ & $\begin{array}{r}\text { SRS pre-op: pain } \\
4.7, \\
\text { self-image 4.1, fxn } \\
4.7 \text {; SRS post-op: } \\
\text { pain } 4.3 \text {, self- } \\
\text { image } 4.4 \text {, fxn } 4.3\end{array}$ & $\begin{array}{l}\text { Durotomy }(\mathrm{n}=1) \text {, pseudarthrosis } \\
(\mathrm{n}=1) \text {, implant failure }(\mathrm{n}=1)\end{array}$ & \\
\hline 2008_Sasso & $\begin{array}{r}\text { Posterior-only }(\mathrm{n}=8) \text {, Anterior- } \\
\text { posterior same-day surgery }(\mathrm{n}=17)\end{array}$ & $\begin{array}{l}100 \% \text { fusion, no } \\
\text { increase in slip } \\
\text { grade or angle. }\end{array}$ & $\begin{array}{l}\text { No permanent neurologic deficits or deterioration; } \\
\text { transient radiculitis } 1 \text {-month post-op }(\mathrm{n}=1)\end{array}$ & $\begin{array}{r}\text { SRS: Extremely or } \\
\text { somewhat satis- } \\
\text { fied (n=24). } \\
\text { Pain: } 8.2 \text { pre-op, } \\
3.4 \text { post-op }\end{array}$ & $\begin{array}{r}\text { Hardware removal due to promi- } \\
\text { nence after fusion achieved }(\mathrm{n}=1) ; \\
\text { equivalent } \mathrm{EBL}\end{array}$ & $\begin{array}{l}\text { This technique offers excellent fusion results, } \\
\text { good clinical outcomes, and prevents further sagit- } \\
\text { tal translation and lumbosacral kyphosis progres- } \\
\text { sion. }\end{array}$ \\
\hline
\end{tabular}

Downloaded from http://ijssurgery.com/ by guest on April 26, 2023 


\begin{tabular}{|c|c|c|c|c|c|c|}
\hline 2009_Hresko & $\begin{array}{r}\text { Posterolateral }(\mathrm{n}=21), \text { Posterolater- } \\
\mathrm{al}+\text { anterior discectomy/interbody } \\
\text { fusion }(\mathrm{n}=5)\end{array}$ & $\begin{array}{r}\text { Grade } \mathrm{A} \text { (definite } \\
\text { solid fusion) } \\
(\mathrm{n}=22) ; \text { Grade } \mathrm{B} \\
\text { (probable solid fu- } \\
\text { sion) }(\mathrm{n}=4)\end{array}$ & $\mathrm{NA}$ & $\mathrm{NA}$ & $\begin{array}{r}\text { Re-operation }(\mathrm{n}=5) \text {, loss of L4-L5 } \\
\text { motor strength }(\mathrm{n}=1) \text {, removal } \\
\text { prominence }(\mathrm{n}=1), \text { revision due to } \\
\text { instrument failure }(\mathrm{n}=3) \text {, transient } \\
\text { neurapraxia }(\mathrm{n}=6) \text {, permanent unilat- } \\
\text { eral L5 weakness }(\mathrm{n}=1)\end{array}$ & $\begin{array}{l}\text { No correlation between improvement in pelvic } \\
\text { version and amount of reduction. Other factors, } \\
\text { such as achievement of solid arthrodesis, may be } \\
\text { more important than reduction of spondylolisthesis } \\
\text { in determining spinopelvic sagittal balance. }\end{array}$ \\
\hline 2010_Sansur & $\begin{array}{r}\mathrm{N}=10,242 \text {; No fusion/ decompres- } \\
\text { sion }(\mathrm{n}=532) \text {, combined ant/post } \\
(\mathrm{n}=893), \text { ant only }(\mathrm{n}=286), \text { post fu- } \\
\text { sion w/o instrument }(\mathrm{n}=491), \text { post } \\
\text { fusion } \mathrm{w} / \text { instrument }(\mathrm{n}=4117), \\
\text { TLIF/PLIF }(\mathrm{n}=3860)\end{array}$ & $\mathrm{NA}$ & $\begin{array}{r}\text { The rate of neurological complications was highest } \\
\text { in the decompression/no fusion group, but it was } \\
\text { not significantly different from other groups } \\
\qquad(\mathrm{p}=0.10)\end{array}$ & NA & $\begin{array}{r}9.2 \% \text { complications rate } \\
\text { Most common complications = dural } \\
\text { tear }(2 \%), \text { infection }(2 \%), \text { neurolog- } \\
\text { ic }(1.2 \%), \text { implant-related }(0.7 \%)\end{array}$ & $\begin{array}{l}\text { Grade level and age but not surgical approach and } \\
\text { history of previous surgery significantly correlated } \\
\text { with increased complication rates. }\end{array}$ \\
\hline \multirow{2}{*}{ 2011_Jalanko } & $\begin{array}{r}\text { HGS in children } \\
(<12.5 \text { years old for females; }<14.5 \\
\text { years old for males })\end{array}$ & $\begin{array}{r}\text { Non-unions but } \\
\text { no effect on long- } \\
\text { term outcome } \\
(\mathrm{n}=5)\end{array}$ & $\begin{array}{r}\text { Posture/gait abnormality }(\mathrm{n}=10), \text { SRA positive } \\
\qquad(\mathrm{n}=14), \text { scoliosis }(\mathrm{n}=8)\end{array}$ & $\begin{array}{r}\text { At final follow-up: } \\
\text { SRS- } 24=92, \\
\text { ODI }=4.5 \%, \\
\text { VAS }=9 \mathrm{~mm}\end{array}$ & $\begin{array}{l}\text { Revision due to nonunion }(\mathrm{n}=3) \text {, } \\
\text { Transient } \mathrm{L} \text { 5 paresis }(\mathrm{n}=1)\end{array}$ & \multirow{2}{*}{$\begin{array}{l}\text { Recommend circumferential in situ fusion for } \\
\text { high-grade slips; spinal fusion can be carried out at } \\
\text { an early age for HGS with good long-term clinical, } \\
\text { functional, radiographic and health-related quality- } \\
\text { of-life outcomes. }\end{array}$} \\
\hline & HGS in adolescents & $\begin{array}{r}\text { Non-unions but } \\
\text { no effect on long- } \\
\text { term outcome } \\
(\mathrm{n}=3)\end{array}$ & $\begin{array}{l}\text { Posture/gait abnormality ( } \mathrm{n}=1) \text {, SRA positive } \\
\qquad(\mathrm{n}=5) \text {, scoliosis }(\mathrm{n}=4)\end{array}$ & $\begin{array}{r}\text { At final follow-up: } \\
\text { SRS-24=94, } \\
\text { ODI }=8.7 \%, \\
\text { VAS }=25.1 \mathrm{~mm}\end{array}$ & $\begin{array}{r}\text { Revision due to nonunion }(\mathrm{n}=1), \mathrm{S} 1 \\
\text { root decompression }(\mathrm{n}=2), \text { wound } \\
\text { infection }(\mathrm{n}-1)\end{array}$ & \\
\hline \multirow{2}{*}{ 2012_Kasliwal } & Pediatric ( $\leq 18$ years old) & $\mathrm{NA}$ & $\begin{array}{r}\text { Nerve root }(\mathrm{n}=9) \text {, cauda equina }(\mathrm{n}=2) \text {, lumbar } \\
\text { nerve palsy }(\mathrm{n}=2) \text {, peroneal n. palsy }(\mathrm{n}=2)\end{array}$ & NA & $\begin{array}{r}24 \% \text { of total patients had a compli- } \\
\text { cation }\end{array}$ & \multirow{2}{*}{$\begin{array}{l}\text { Osteotomy was the only surgical predictor of neu- } \\
\text { rologic deficit; new neurologic deficit post-op did } \\
\text { not correlate to decompression, reduction or revi- } \\
\text { sion surgery }\end{array}$} \\
\hline & Adults ( $>18$ years old) & NA & $\begin{array}{r}\text { Nerve root }(n=8) \text {, cauda equina }(n=0) \text {, lumbar } \\
\text { nerve. palsy }(n=1) \text {, peroneal nerve. palsy }(n=0)\end{array}$ & NA & $\begin{array}{r}26 \% \text { of total patients had a compli- } \\
\text { cation }\end{array}$ & \\
\hline
\end{tabular}


Although no definitive recommendations can be established, literature suggests that circumferential fusion with interbody support has lower incidence of pseudarthrosis and possibly better chance of longterm clinical success compared to those treated with posterior only techniques in the absense of interbody support. Despite this finding, relative to additional interbody support from a posterior-based approach (TLIF and PLIF), combined anterior/posterior approaches are associated with longer hospital stays and increased blood loss and surgical time, ${ }^{32,33}$ which can be associated with greater risk of complications. ${ }^{34}$ DeWald et al. reviewed adult HGS patients and recommended in situ posterior fusion with instrumentation from L4 to S1 with consideration for partial reduction and vertebrectomy. Helenius et al. retrospectively studied the pediatric population undergoing posterolateral, anterior and circumferential fusion in situ without instrumentation and found that the circumferential group had the best health-relatedquality-of-life scores and lowest percentage of lumbosacral kyphosis progression. No similar adult studies were found. Nonetheless, well-designed prospective studies that directly compare different fusion modalities are necessary to provide better evidence on comparative treatment effectiveness.

Surgical management of HGS involves various techniques such as instrumentations, reductions, and decompressions. Instrumentation plays a more significant role in adults than children as uninstrumented in situ circumferential fusion is considered more viable in treating pediatric HGS. ${ }^{6,8,17}$ With regards to the extent of construct, instrumentation to the L4-5 level provides better stability and more vertical fusion as opposed to the more horizontal fusion that occurs with L5-sacrum fusion. ${ }^{35}$ Some surgeons extend instrumentation to the ilium due to high failure rates at the lumbosacral juction with long posterior instrumentation ending at $\mathrm{S} 1,{ }^{36}$ but its value for HGS patients remains questionable. ${ }^{16,20}$

Innovative technology such as the Luque box and the Jackson intrasacral rod have shown improved fusion and clinical outcomes but have also resulted in higher complication rates attributed to the instrumentation itself. ${ }^{22}$ Although technological advancement is paramount to providing patients with the best possible care, the importance of properly evaluating novel spinal instrumentation is equally critical prior to routine clinical acceptance. Two LOE III studies discussed uninstrumented fusion in children. ${ }^{8,17}$ Poussa et al. showed that compared to those treated with reduction and instrumented fusion with transpedicular fixation, adolescents who were fused in situ circumferentially without instrumentation had better ODI and SRS scores despite having evidence of asymptomatic nerve root impingement on MRI and no reduction of their slip grade. ${ }^{8}$ Molinari et al. attempted uninstrumented in situ PLF in 11 pediatric patients and reported a $45 \%$ pseudarthrosis rate $(n=5)^{17}$ with pain and increased deformity requiring reoperations. These patients underwent circumferential fusions with instrumentation and ultimately achieved solid fusion.

Various pedicle screw constructs, extending from L3, L4, or L5 to S1 with or without iliac fixation, and transsacral screws were used for HGS procedures. The most common instrumentation for adults were L4-S1 pedicle screws. ${ }^{8,16,20,21,23}$ For children, reduced circumferential monosegmental L5-S1 instrumentation and circumferential in situ fusion from L4/L5 to $\mathrm{S} 1$ without instrumentation yielded the best results with no neurological complications and improvement of slip angle. ${ }^{18}$ One LOE III study found similar complication rates and surgical outcomes in unilateral TLIF's and transvertebral screw fixations of the lumbosacral spine. ${ }^{20}$ Two LOE IV studies reported a subcohort receiving instrumentation to the ilium without report on results, ${ }^{16}$ and one study, without reference to evidence, recommended extension to the ilium in adult patients, particularly for revisions and unstable spondylolisthesis. ${ }^{23}$

A LOE IV study investigating two constructs, a Luque box and a Jackson intrasacral rod extending from L4 to sacrum, reported that all patients achieved solid fusion with an overall 19\% reoperation rate and a $23 \%$ incidence of transient neurological deficits. ${ }^{22}$ One LOE IV study investigating the "double-plate" technique found that it caused implant-related complications in $22 \%$ of patients, late infections in $12 \%$, and postoperative neurological complications in $30 \%$, despite achieving optimal deformity reduction and high fusion rates. ${ }^{19}$ 
Nine clinical studies reported on decompression but none directly-examined it as an independent variable. Gill decompression, involving the removal of L5 lamina and fibrocartilaginous tissue at the pars, was most commonly performed. Poussa et al. reported better outcomes in reduction with decompression than in in situ fusion with no decompression (decompression is not concurrently performed with in situ fusions). ${ }^{8}$ This difference could not be attributed to decompression alone, given the group disparity and the multiple factors being compared.

\section{Reduction of Slip Angle/Grade}

Two LOE III studies compared reduced and nonreduced cohorts. ${ }^{8,17}$ Poussa et al. found that the adolescent in situ group had better ODI and SRS scores than the reduced cohort. ${ }^{8}$ Further, this group found that patients treated with partial reduction had better clinical outcomes - mean ODI was 7.2 in partial reduction vs. 1.6 in in situ group and SRS score 90.0 vs. 103.9. ${ }^{12}$ Molinari et al. revealed increased pseudarthrosis and deformity progression in in situ PLF group compared to the reduced PLF group (45\% vs. $29 \%, 36 \%$ vs. $29 \%$, respectively). ${ }^{17}$ Dewald et al. was the only study addressing in situ fusion and reduction in adults, but there was not enough evidence to reach definitive conclusions. ${ }^{23}$ One LOE III and one LOE IV study reported cases of attempts to reduce slip angle by operative positioning without attempting to change slip grade. ${ }^{20,21}$ Both studies reported slip angle improvements of $10-15^{\circ}$ without significant slip grade change, safe and effective surgical results with stable arthrodesis rate, no long-term neurologic deficits, and improvements in gait disturbance. ${ }^{20,21}$ No studies directly compared partial and complete reduction. Three LOE III and two LOE IV studies reported on partial slip reduction techniques, either via temporary distraction or direction reduction. ${ }^{8,16-18,23}$ Two studies incorporated decompression and partial reduction techniques and highlighted that in situ may be associated with improved outcomes in children and adolescents but only with anterior support. This observation is due to concerns about increased incidence of pseudarthrosis and deformity progression without anterior support. ${ }^{8,17}$ One LOE IV study attempted complete reduction in all patients and achieved full reduction in $95 \%$ of patients. ${ }^{19}$ However, they reported poor outcomes overall and a high incidence of neurological and infectious complications. Another study reported complete reduction only in two patients who had abnormally mobile olistheses, but no separate sub-cohort results were reported. ${ }^{23}$

The extent of reduction and its clinical relevancy and safety are controversial, as reports of associated neurologic complication rates in the literature are mixed. Molinari et al. reported positive outcomes in a circumferential partial reduction cohort, but results were not significantly different from the in situ cohort, implying that reduction was not the most important contributor to positive outcome. ${ }^{17<}$ Poussa et al. stated that in situ circumferential fusion is preferable for pediatric patients over reduction. However, partial reduction allows sagittal malalignment correction and would theoretically create a more favorable environment for fusion by increasing contact surface area between two vertebrae and thus increasing osteogenic, osteoinductive and osteoconductive effects. Studies indicate successful clinical outcome with slip angle reduction without an increased risk for neurological complications, but definitive comparative data on slip angle reduction without surgical reduction is lacking. ${ }^{19,20}$

\section{Post-Operative Management}

Only one of the twenty studies reported on post operative management of in situ fusions. ${ }^{17}$ Patients were immobilized for 4 to 7 months in a hyperextension cast incorporating both thighs. In comparison, patients treated with instrumentation had 4 months of immobilization after surgery that consisted of bed rest. In addition to bed rest, some patients also wore a cast or a brace.

\section{Conclusion/Limitations}

This systematic review presents potential risk factors for slip progression and radiographic and clinical outcomes based on different types of surgical procedure. There is strong evidence that treatment of HGS should be influenced based on pelvic parameters, i.e. sacral slope and pelvit tilt that determine pelvic balance. Unfortunately, prospective, randomized and controlled studies providing a more reliable conclusion on surgical treatment strategies for HGS have 
not been conducted to date. Surgeons must weigh fixing the deformity with avoiding complications, assessing patient satisfaction and patients' understanding of possible complications.

\section{Evidence Summary}

Overall strength of effectiveness of various surgical procedures for HGS is "low," meaning there is low confidence that the evidence reflects true effect and further research is likely to change the confidence in the estimate of effect. ${ }^{37}$ The overall strength of appropriateness of reduction and instrumentation in addition to fusion for HGS is "low," but "insuffcient" for decompression, meaning that evidence is either unavailable or does not permit a conclusion. The overall strength of correlation between higher PI and progression is "high." For adults, this review recommends that instrumented circumferential fusion provides the most promising clinical and radiographic outcome compared to anterior-only fusion and PLF in adults. For children and adolescents, this review recommends in situ fusion along with thorough long-term follow-up to check for deformity and quality of life. Full details are available in Table 7.

\section{References}

1. Pizzutillo PD, Mirenda W, MacEwen GD. Posterolateral fusion for spondylolisthesis in adolescence. Journal of Pediatric Orthopaedics 1986;6:311-6.

2. Transfeldt EE, Mehbod AA. Evidence-based medicine analysis of isthmic spondylolisthesis treatment including reduction versus fusion in situ for high-grade slips. Spine 2007;32:S126-S9.

3. Wright JG, Swiontkowski MF, Heckman JD. Introducing levels of evidence to the journal. The Journal of Bone \& Joint Surgery 2003;85:1-3.

4. Atkins D, Eccles M, Flottorp S, et al. Systems for grading the quality of evidence and the strength of recommendations I: critical appraisal of existing approaches The GRADE Working Group. BMC Health Services Research 2004;4:38.

5. West SL, King V, Carey TS, et al. Systems to rate the strength of scientific evidence: Agency for Healthcare Research and Quality, US Department of Health and Human Services; 2002.<
6. Jalanko T, Helenius I, Remes V, et al. Operative treatment of isthmic spondylolisthesis in children: a long-term, retrospective comparative study with matched cohorts. European Spine Journal 2011;20:766-75.

7. Helenius I, Lamberg T, Österman K, et al. Posterolateral, anterior, or circumferential fusion in situ for high-grade spondylolisthesis in young patients: a long-term evaluation using the Scoliosis Research Society questionnaire. Spine 2006;31:190-6.

8. Poussa M, Remes V, Lamberg T, et al. Treatment of severe spondylolisthesis in adolescence with reduction or fusion in situ: long-term clinical, radiologic, and functional outcome. Spine 2006;31:583-90. 9. Huang RP, Bohlman HH, Thompson GH, PoeKochert C. Predictive value of pelvic incidence in progression of spondylolisthesis. Spine 2003;28:2381-5.

10. Curylo LJ, Edwards C, DeWald RW. Radiographic markers in spondyloptosis: implications for spondylolisthesis progression. Spine 2002;27:2021-5. 11. Hanson DS, Bridwell KH, Rhee JM, Lenke LG. Correlation of pelvic incidence with low-and highgrade isthmic spondylolisthesis. Spine 2002;27:2026-9.

12. Hresko MT, Labelle H, Roussouly P, Berthonnaud E. Classification of high-grade spondylolistheses based on pelvic version and spine balance: possible rationale for reduction. Spine 2007;32:2208-13. 13. Vialle R, Ilharreborde B, Dauzac C, Lenoir T, Rillardon L, Guigui P. Is there a sagittal imbalance of the spine in isthmic spondylolisthesis? A correlation study. European Spine Journal 2007;16:1641-9. 14. Vialle R, Schmit P, Dauzac C, Wicart P, Glorion C, Guigui P. Radiological assessment of lumbosacral dystrophic changes in high-grade spondylolisthesis. Skeletal radiology 2005;34:528-35.

15. Labelle H, Roussouly P, Chopin D, Berthonnaud E, Hresko T, O'Brien M. Spino-pelvic alignment after surgical correction for developmental spondylolisthesis. European spine journal : official publication of the European Spine Society, the European Spinal Deformity Society, and the European Section of the Cervical Spine Research Society 2008;17:1170-6.

16. Hanson DS, Bridwell KH, Rhee JM, Lenke LG. Dowel fibular strut grafts for high-grade dysplastic 
isthmic spondylolisthesis. Spine 2002;27:1982-8.

17. Molinari RW, Bridwell KH, Lenke LG, Baldus

C. Anterior column support in surgery for high-

grade, isthmic spondylolisthesis. Clinical or-

thopaedics and related research 2002;394:109-20.

18. Shufflebarger HL, Geck MJ. High-grade isthmic

dysplastic spondylolisthesis: monosegmental surgical treatment. Spine 2005;30:S42-S8.

19. Vialle R, Charosky $S$, Padovani J-P, Rigault $P$, Glorion C. Surgical treatment of high-grade lumbosacral spondylolisthesis in childhood, adolescent and young adult by the "double-plate" technique: a past experience. European Spine Journal 2006;15:1210-8

Table 7. Strength of Evidence.

Baseline strength: HIGH=majority of articles Level I/II LOW=majority of articles Level III/IV

Upgrade: Large magnitude of effect and/or dose response gradient

Downgrade: Inconsistency of results, indirectness of evidence, imprecision of effect estimates

$$
\begin{array}{r}
\text { Strength } \\
\text { of evi- } \\
\text { dence }
\end{array}
$$

Question 1: What is the effectiveness of the various surgical approaches for HGS?

\begin{tabular}{|c|c|c|}
\hline Reduction & Low & $\begin{array}{l}\text { Overall, the evidence on the incorporation of reduction is inconsistent. Three LOE III and two LOE } \\
\text { IV clinical studies reported negative clinical outcome in partial reduction group with worse ODI and } \\
\text { SRS scores but positive radiographic outcome with less pseudarthrosis and deformity progression } \\
\text { on reduction of slip angle and slip grade. One LOE IV study reported full reduction in } 95 \% \text { of pa- } \\
\text { tients undergoing complete reduction but had high complication rate. For children and adolescents } \\
\text { in situ fusion with long-term follow-up is recommended }\end{array}$ \\
\hline Instrumentation & Low & $\begin{array}{l}\text { No studies used instrumentation as the sole primary variable. Most studies included instrumentation } \\
\text { with varying surgical approaches or other additional surgical maneuvers such as reduction. Use o } \\
\text { instrumentation is dependent on surgical approach and surgeon preference. Luque box, Jackson in } \\
\text { trasacral rod and double-plate technique are not recommended }\end{array}$ \\
\hline Decompression & Insufficient & $\begin{array}{l}\text { There is no study directly examining decompression as an independent variable. Three Level III Ev- } \\
\text { idence studies performing in situ fusion without decompression reported good clinical and radi } \\
\text { ographic outcome. Higher-level studies reporting on decompression are necessary for a definitive } \\
\text { conclusion }\end{array}$ \\
\hline
\end{tabular}

\begin{tabular}{|l|l|l|l|l}
\hline $\begin{array}{l}\text { Clinical outcome } \\
\text { (Pain, function, } \\
\text { etc.) }\end{array}$ & Low & $\begin{array}{r}\text { Three LOE III and one LOE IV retrospective cohort studies reported good outcomes for function } \\
\text { pain, and satisfaction in patients with solid fusion regardless of the surgical procedure, but circum- } \\
\text { ferential fusion provided the best clinical outcome results }\end{array}$ \\
\hline $\begin{array}{l}\text { Radiographic } \\
\text { outcome }\end{array}$ & Low & $\begin{array}{r}\text { One LOE III and two LOE IV retrospective cohort studies reported no difference in fusion rate, but } \\
\text { two Level III Evidence and one Level IV Evidence studies reported significantly higher } \\
\text { pseudarthrosis rate in posterior-only procedures }\end{array}$ \\
\hline
\end{tabular}

Question 2: What is the effectiveness of additional surgical maneuvers for HGS?

\begin{tabular}{|c|c|c|c|c|c|}
\hline $\begin{array}{l}\text { Circumferential } \\
\text { vs. Posterior- } \\
\text { only vs. } \\
\text { Anterior-only }\end{array}$ & Low & $\begin{array}{l}\text { Three Level III Evidence and one Level IV Evidence retrospective cohort studies report no long- } \\
\text { term difference in complications. Operation time and hospital stay was slightly higher in circumfer- } \\
\text { ential procedures. One Level IV Evidence retrospective study reported higher operative blood loss } \\
\text { in PLIF compared to ALIF, but outcomes were similar. }\end{array}$ & LOW & NO & NO \\
\hline \multicolumn{6}{|c|}{ Question 4: Who is at risk for progression to higher grade spondylolisthesis? } \\
\hline Pelvic Incidence & $\mathrm{HIGH}$ & Two Level II Evidence studies indicated no correlation between higher PI and progression. & $\mathrm{HIGH}$ & NO & NO \\
\hline Sacral slope & HIGH & $\begin{array}{l}\text { Two Level II Evidence studies indicated no correlation between higher SS and progression. They } \\
\text { reported that higher PI controls have high PT and SS and that SS may be predictive of reducibility. }\end{array}$ & HIGH & NO & NO \\
\hline $\begin{array}{l}\text { Age and grade } \\
\text { of spondylolis- } \\
\text { thesis }\end{array}$ & HIGH & $\begin{array}{r}\text { One Level II Evidence study indicated positive correlation between higher PI, age and grade of } \\
\text { spondylolisthesis but not of progression. }\end{array}$ & HIGH & NO & NO \\
\hline
\end{tabular}

Question 3: What is the comparative safety of the various surgical approaches for HGS?

Downloaded from http://ijssurgery.com/ by guest on April 26, 2023 
20. Rodriguez-Olaverri JC, Zimick NC, Merola A, et al. Comparing the clinical and radiological outcomes of pedicular transvertebral screw fixation of the lumbosacral spine in spondylolisthesis versus unilateral transforaminal lumbar interbody fusion (TLIF) with posterior fixation using anterior cages. Spine 2008;33:1977-81.

21. Sasso RC, Shively KD, Reilly TM. Transvertebral transsacral strut grafting for high-grade isthmic spondylolisthesis L5-S1 with fibular allograft. Journal of spinal disorders \& techniques 2008;21:328-33.

22. Hresko MT, Hirschfeld R, Buerk AA, Zurakowski $\mathrm{D}$. The effect of reduction and instrumentation of spondylolisthesis on spinopelvic sagittal alignment. Journal of Pediatric Orthopaedics 2009;29:157-62.

23. DeWald CJ, Vartabedian JE, Rodts MF, Hammerberg KW. Evaluation and management of highgrade spondylolisthesis in adults. Spine 2005;30:S49-S59.

24. Sansur CA, Reames DL, Smith JS, et al. Morbidity and mortality in the surgical treatment of 10,242 adults with spondylolisthesis. Journal of Neurosurgery: Spine 2010;13:589-93.

25. Kasliwal MK, Smith JS, Shaffrey CI, et al. Short-term Complications Associated With Surgery for High-Grade Spondylolisthesis in Adults and Pediatric Patients: A Report From the Scoliosis Research Society Morbidity and Mortality Database. Neurosurgery 2012;71:109-16.

26. Legaye J, Duval-Beaupere G, Hecquet J, Marty C. Pelvic incidence: a fundamental pelvic parameter for three-dimensional regulation of spinal sagittal curves. European Spine Journal 1998;7:99-103.

27. Vialle R, Dauzac C, Khouri N, Wicart P, Glorion C, Guigui P. Sacral and lumbar-pelvic morphology in high-grade spondylolisthesis. Orthopedics 2007;30:642-9.

28. Li Y, Hresko MT. Radiographic analysis of spondylolisthesis and sagittal spinopelvic deformity. The Journal of the American Academy of Orthopaedic Surgeons 2012;20:194-205.

29. Schwab FJ, Lafage V, Farcy J-P, Bridwell KH, Glassman S, Shainline MR. Predicting outcome and complications in the surgical treatment of adult scoliosis. Spine 2008;33:2243-7.

30. Lafage V, Schwab F, Skalli W, et al. Standing balance and sagittal plane spinal deformity: analysis of spinopelvic and gravity line parameters. Spine 2008;33:1572-8.

31. Schuller S, Charles YP, Steib J-P. Sagittal spinopelvic alignment and body mass index in patients with degenerative spondylolisthesis. European Spine Journal 2011;20:713-9.

32. Dorward IG, Lenke LG, Bridwell KH, et al. Transforaminal versus Anterior Lumbar Interbody Fusion in Long Deformity Constructs: a matched cohort analysis. Spine 2013;38:E755-E62.

33. Freudenberger C, Lindley EM, Beard DW, et al. Posterior versus anterior lumbar interbody fusion with anterior tension band plating: retrospective analysis. Orthopedics 2009;32:492.

34. Hee HT, Castro Jr FP, Majd ME, Holt RT, Myers L. Anterior/posterior lumbar fusion versus transforaminal lumbar interbody fusion: analysis of complications and predictive factors. Journal of spinal disorders \& techniques 2001;14:533-40.

35. Lamartina C, Zavatsky JM, Petruzzi M, Specchia N. Novel concepts in the evaluation and treatment of high-dysplastic spondylolisthesis. European Spine Journal 2009;18:133-42.

36. Cunningham BW, Sefter JC, Hu N, Kim SW, Bridwell KH, McAfee PC. Biomechanical comparison of iliac screws versus interbody femoral ring allograft on lumbosacral kinematics and sacral screw strain. Spine 2010;35:E198-E205.

37. Norvell DC, Dettori JR, Skelly AC, Riew KD, Chapman JR, Anderson PA. Methodology for the Systematic Reviews on an Adjacent Segment Pathology. Spine 2012;37:S10-S7.

38. Wang Z, Wang B, Yin B, Liu W, Yang F, Lv G. The relationship between spinopelvic parameters and clinical symptoms of severe isthmic spondylolisthesis: a prospective study of 64 patients. Eur Spine J. 2014;23(3):560-8. doi:10.1007/ s00586-013-3064-2.

\section{Disclosures}

Virginie LaFage owns stock in Nemaris Inc and is on its Board of Directors; has speaking and/or teaching arrangements with MSD, DePuy, and Medicrea; and has received grants from DePuy, ISSG, SRS, and the NIH. The other authors report no disclosures. 


\section{Corresponding Author}

Peter G. Passias, M.D. New York Spine Institute. 361

Merrick Avenue, Westbury, New York 11590. Pe-

ter.Passias@nyumc.org.
Published 1 October 2015.

This manuscript is generously published free of charge by ISASS, the International Society for the Advancement of Spine Surgery. Copyright $\odot 2015$ ISASS. To see more or order reprints or permissions, see http://ijssurgery.com. 